Document downloaded from:

http://hdl.handle.net/10251/111959

This paper must be cited as:

Payri González, F.; Arnau Martínez, FJ.; Piqueras Cabrera, P.; Ruiz Lucas, MJ. (2018). Lumped Approach for Flow-Through and Wall-Flow Monolithic Reactors Modelling for RealTime Automotive Applications. SAE Technical Papers. doi:10.4271/2018-01-0954

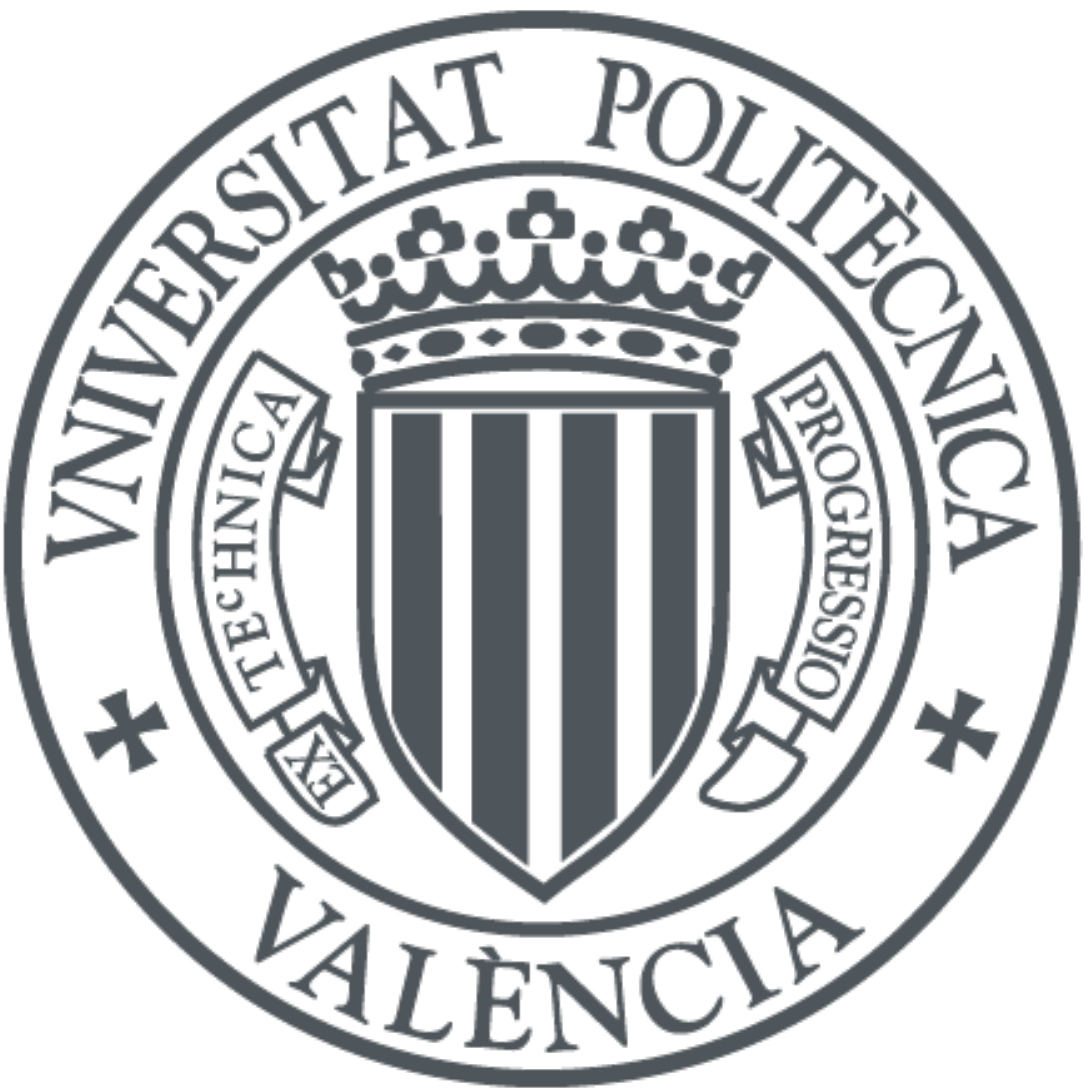

The final publication is available at

http://doi.org/10.4271/2018-01-0954

Copyright SAE Internacional

Additional Information 


\title{
Lumped Approach for Flow-Through and Wall-Flow Monolithic Reactors Modelling for Real-Time Automotive Applications
}

\author{
Francisco Payri, Francisco José Arnau, Pedro Piqueras, María José Ruiz
}

\begin{abstract}
The increasingly restrictive legislation on pollutant emissions is involving new homologation procedures driven to be representative of real driving emissions. This context demands an update of the modelling tools leading to an accurate assessment of the engine and aftertreatment systems performance at the same time as these complex systems are understood as a single element. In addition, virtual engine models must retain the accuracy while reducing the computational effort to get closer to real-time computation. It makes them useful for pre-design and calibration but also potentially applicable to on-board diagnostics purposes. This paper responds to these requirements presenting a lumped modelling approach for the simulation of aftertreament systems. The basic principles of operation of flow-through and wall-flow monoliths are covered leading the focus to the modelling of gaseous emissions conversion efficiency and particulate matter abatement, i.e. filtration and regeneration processes. The model concept is completed with the solution of pressure drop and heat transfer processes. The lumped approach hypotheses and the solution of the governing equations for every submodel are detailed. While inertial pressure drop contributions are computed from the characteristic pressure drop coefficient, the porous medium effects in wall-flow monoliths are considered separately. Heat transfer sub-model applies a nodal approach to account for heat exchange and thermal inertia of the monolith substrate and the external canning. In wall-flow monoliths, the filtration and porous media properties are computed as a function of soot load applying a spherical packed bed approach. The soot oxidation mechanism including adsorption reactant phase is presented. Concerning gaseous emissions, the general scheme to solve the chemical species transport in the bulk gas and washcoat regions is also described. In particular, it is finally applied to the modelling of $\mathrm{CO}$ and $\mathrm{HC}$ abatement in a DOC and DPF brick. The model calibration steps against a set of steady-state in-engine experiments allowing separate certain phenomena are discussed. As a final step, the model performance is assessed against a transient test during which all modelled processes are taking place simultaneously under highly dynamic driving conditions. This test is simulated imposing different integration time-steps to demonstrate the model's potential for real-time applications.
\end{abstract}

\section{Introduction}

Growing concerns about pollution effects on environment and human health is leading worldwide to the adoption of increasingly stringent emission standards [1]. This context has established the aftertreatment systems as essential devices in both spark and compression ignition engines [2], in addition to the research in different but synergistic areas like injection, combustion, boosting systems, EGR or control strategies.
Compression ignition engines require the combination of a wall-flow particulate filter, which collects the particulate matter, with a series of flow-through monolithic reactors in charge for $\mathrm{CO}, \mathrm{HC}$ and $\mathrm{NOx}$ abatement due to the lean-burn combustion requirements of these engines. As a consequence, a great variety of architectures combining several devices is expected in the next years being the particular components conditioned by the specific NOx abatement solution $[3,4]$. On the other hand, the Three-Way Catalyst (TWC) can fulfill the abatement of $\mathrm{CO}, \mathrm{HC}$ and NOx as an only device in spark ignition engines due to the stoichiometric operation conditions. Concerning particulate emissions, direct injection gasoline engines lead to particulate formation [5]. Therefore, these engines require the use of a wall-flow Gasoline Particulate Filter (GPF) to fulfill the regulation limits [6].

In parallel to limits, upcoming standards are widening the boundary conditions under which the engine emissions must be carefully controlled. Thus, besides the driving cycle, European regulations cover durability requirements in form of deterioration factors, low temperature tests, altitude impact or Real Driving Emissions (RDE) assessment constrained by conformity factors. In addition, On-Board Diagnostics (OBD) is also required imposing monitoring requirements related to emission control, in particular on exhaust aftertreatment systems.

This regulatory framework has led to the acknowledgment of the aftertreatment devices as a part of the integral engine concept [7]. Thus, the automotive industry demands flexible aftertreatment modelling tools able to be used in OBD [8] at the same time as the main physical and chemical phenomena are properly treated. This is essential to couple these tools to engine models both for control and gas dynamics [7] applications. The computational models must ensure robustness, feasibility and cost effectiveness as well as a deep understanding of the governing physical and chemical phenomena.

Different approaches for monolithic wall-flow and flow-through devices have been proposed to deal with these objectives. In wallflow monoliths, the Bisset's proposal based on the solution of a single pair of inlet and outlet channels [9] as well as the work of Konstandopoulos and Johnson [10] has served as a basis for the development of new tools mainly driven to reduce the computational effort. Lumped [11], 1D-lumped [12] and 1D [13] model approaches have been discussed in the literature for real-time applications. In the last case, decoupling techniques are applied to the solution procedure in order to reduce the computational effort demanded by traditional 1D models [13]. Similarly, flow-through monolith real-time models have been also proposed, based on 1D [14], use of neural networks [15] and control-oriented [16] approaches.

As a particular response to the need of flexible computational tools for exhaust aftertreatment systems, a classical lumped model is presented in this work. The model formulation for wall-flow and 
flow-through monoliths is described in detail. The model conception is based on a modular approach covering pressure drop, heat transfer and chemical mechanism sub-models is considered. The heat transfer modelling is based on a nodal approach adapted from 1D modelling [17] to account for gas to wall heat exchange, heat losses against environment and thermal inertia of the monolith substrate and the external canning. The abatement of gaseous pollutants is modelled solving the chemical species transport in the bulk gas and washcoat regions assuming quasi-steady flow. In the case of wall-flow monoliths, the filtration efficiency computation is included as basic performance according to the proposal of Serrano et al. [1817]. In these devices, the geometrical properties of the porous medium and the inlet channel geometry are described as a function of the particulate matter load, which varies due to filtration and regeneration and impacts on chemical, thermo- and fluid-dynamic processes. Partial soot penetration into the porous wall is assumed [19]. The soot oxidation mechanism includes the modelling of the adsorption reactant phase on soot particles. The model potential is assessed by means of the modelling of brick composed by a Diesel Oxidation Catalyst (DOC) and a Diesel Particulate Filter (DPF), which was tested in an engine test bench. The calibration methodology for DOC and DPF firstly considers motoring and steady-state operation, which enables the isolation of certain phenomena. As a final step, the model deals with a World harmonized Light vehicles Test Cycle (WLTC) during which all processes take place simultaneously under dynamic driving conditions, hence being suitable for performance and realtime ability evaluation.

\section{Exhaust aftertreatment system lumped model}

The presented exhaust gas aftertreatment models for flow-through and wall-flow monoliths are integrated into a gas dynamics software called Virtual Engine Model (VEMOD), which has been developed at the Research Institute CMT-Motores Térmicos. VEMOD is a computational tool that aims to respond to highly limiting requirements of emission standards imposed by new homologation procedures, closer to real-world driving conditions in terms of engine dynamic operation, ambient temperature and altitude concerns. The current context demands the support of new computational tools able to accurately predict the engine performance and emissions while reducing the cost of expensive tests campaigns usually based on chassis-dyno calibration and road validation.

As sketched in Figure 1, VEMOD deals with these purposes based on an integral engine modelling that covers the calculation of a set of key processes. Firstly, the air management is computed by means of a 1D gas dynamics model [20] which deals with flow properties transport along the intake and exhaust systems as well as the longand short-route EGR paths. Thus, specific sub-models are considered for the boosting system, i.e. compressor [21] and turbine [22], aircharge and EGR coolers, throttle valves, heat transfer, etc. The gas dynamics model is coupled to a cylinder model whose main function is the prediction of the in-cylinder conditions based on the combustion process. A detailed heat transfer model $[23,24]$ is used to obtain the heat rejection to the chamber walls and a mechanical losses model [25] allows predicting the brake power. An emission sub-model is coupled to the combustion process to provide raw $\mathrm{CO}$, $\mathrm{HC}, \mathrm{NOx}$, and soot emissions as a function of the engine operating conditions. Sub-models are also considered for auxiliary systems related to cooling and lubrication functions. Air management and cylinder models set the boundary conditions to evaluate the performance of the different exhaust aftertreatment devices. In the particular case of the compression-ignition engine, DOC, DPF and deNOx systems, i.e. Lean-NOx Trap (LNT) or Selective Catalyst

Page 2 of 22
Reduction (SCR), can be considered. Finally, the engine model is coupled to two additional sub-models providing the capability to simulate driving cycles. On the one hand, a control system model emulates the electronic control unit (ECU) of the engine. The ECU sets different engine actuators, as the throttle position, the injection settings, the exhaust gas recirculation valves, the VGT position, etc., based on engine sensors data. In particular, the throttle demand is imposed by the driver that follows the driving cycle. It is simulated under the constraint of a particular vehicle model, which determines the engine speed as main input for the engine model.

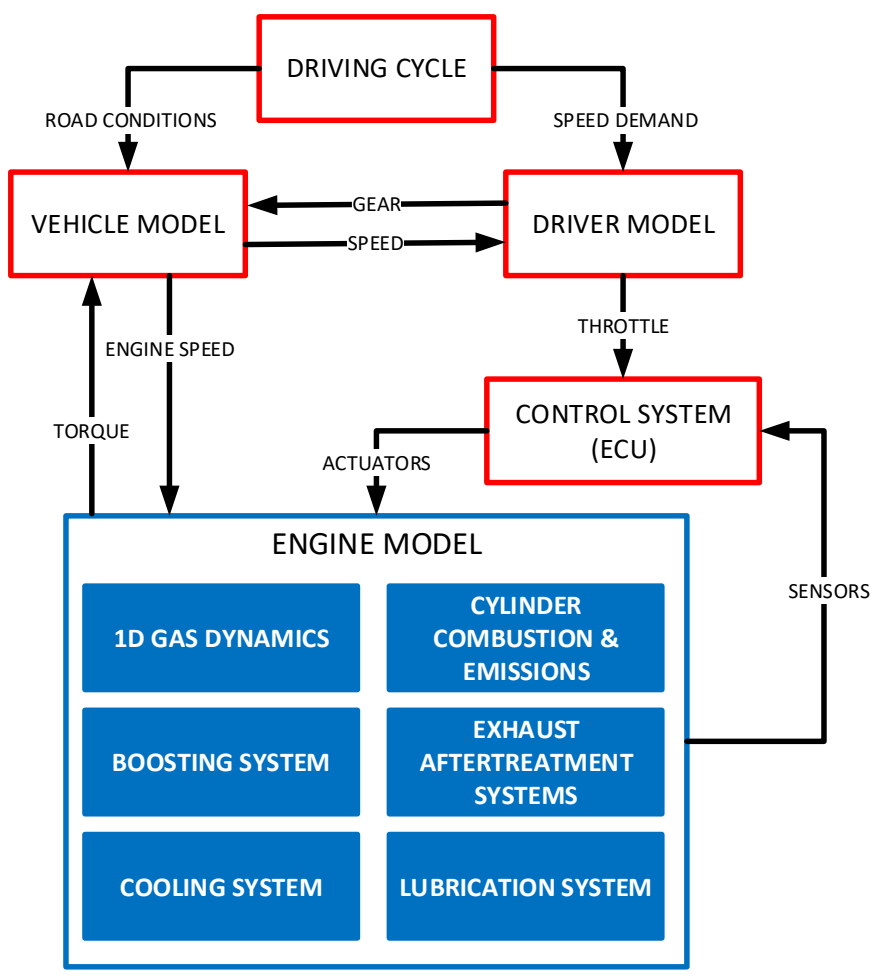

Figure 1. Flow-chart of Virtual Engine Model (VEMOD) modules.

The definition of the exhaust aftertreatment systems model is based on a decoupled lumped quasi-steady approach that ensures the modularity for its integration in different computational environments. Its use in gas dynamics codes implies that the exhaust aftertreatment models are used as boundary conditions between the end cells of two 1D elements (ducts). In this case, the thermodynamic properties are imposed at the inlet (pressure, temperature and composition) and outlet (pressure) of the flow-through or wall-flow device, as presented in Figure 2 and Figure 3. The lumped model solution provides the fluxes between these adjacent cells at time $t$, which are necessary to solve the flow properties at the end of the time step $(t+\Delta t)$. In the case of standalone execution of the models, for example as embedded software in OBD applications, the exhaust mass flow might be imposed to the lumped model.

The flow-through monolith model deals with the main physical and chemical processes to determine the performance of the system in fluid-dynamic and emissions terms. It is comprised by three submodels solving pressure drop, heat transfer and chemical reactions. As a lumped model, constant flow properties are assumed along the monolith length. The model provides a lumped description of the monolith substrate properties and the prediction of the flow properties at the monolith outlet. According to the flow-chart shown in Figure 2, the model is able to predict mass flow across the 
monolith. It is calculated by the pressure drop sub-model. The outlet gas composition, i.e. pollutants conversion efficiency, is computed by the chemical reactions modelling. Finally, the outlet gas temperature as well as the substrate and external canning wall temperature are determined by the heat transfer and the heat released in the chemical reactions. To do that, the main input data are the inlet gas temperature and the composition. As previously stated, the inlet and output pressure are provided in gas dynamics software for mass flow prediction. On the contrary, the on-board vehicle applications might use exhaust mass flow together with the inlet temperature to provide a pressure drop estimate.

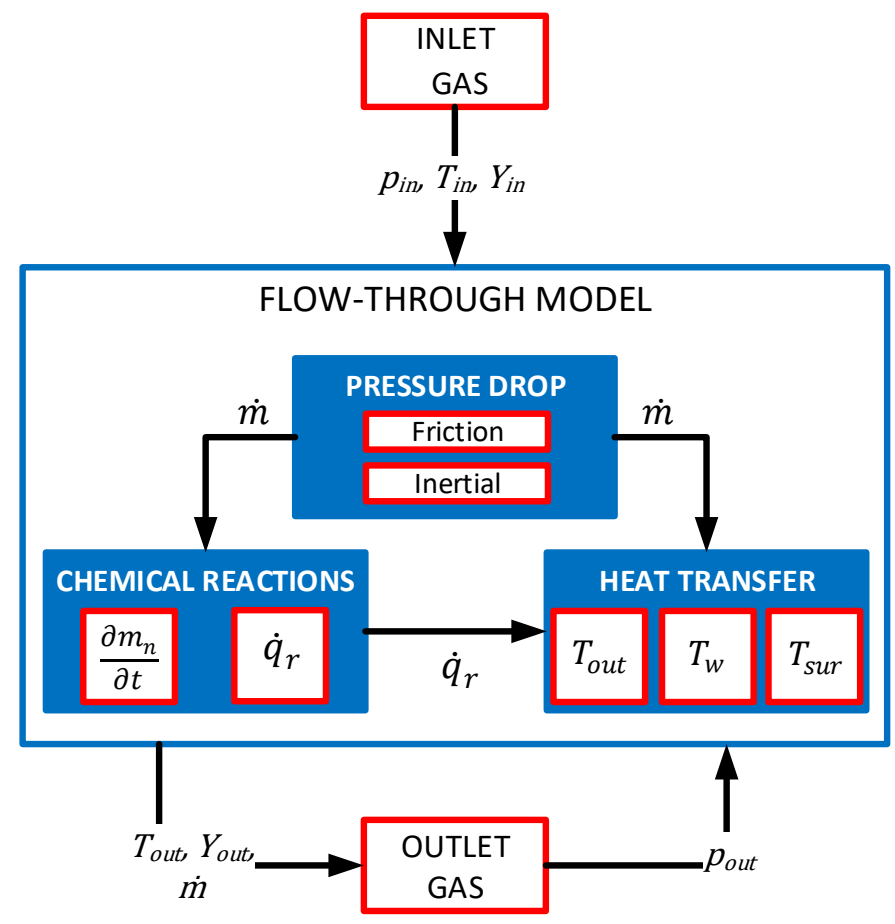

Figure 2. Flow-chart of flow-through lumped model.

The wall-flow monolith model is structured in the same way. The pressure drop, the inlet gas temperature and the composition are the input flow properties for the model solution in gas dynamics software environment. Figure 3 shows the main interactions between the different sub-models. Due to the operation principle concerning particulate matter collection, this model includes filtration and soot oxidation sub-models. In turn, these processes, as well as a correct mass flow prediction from pressure drop, demand a porous medium sub-model to account for the variation in micro- and meso-geometry of the substrate and the inlet channels respectively.

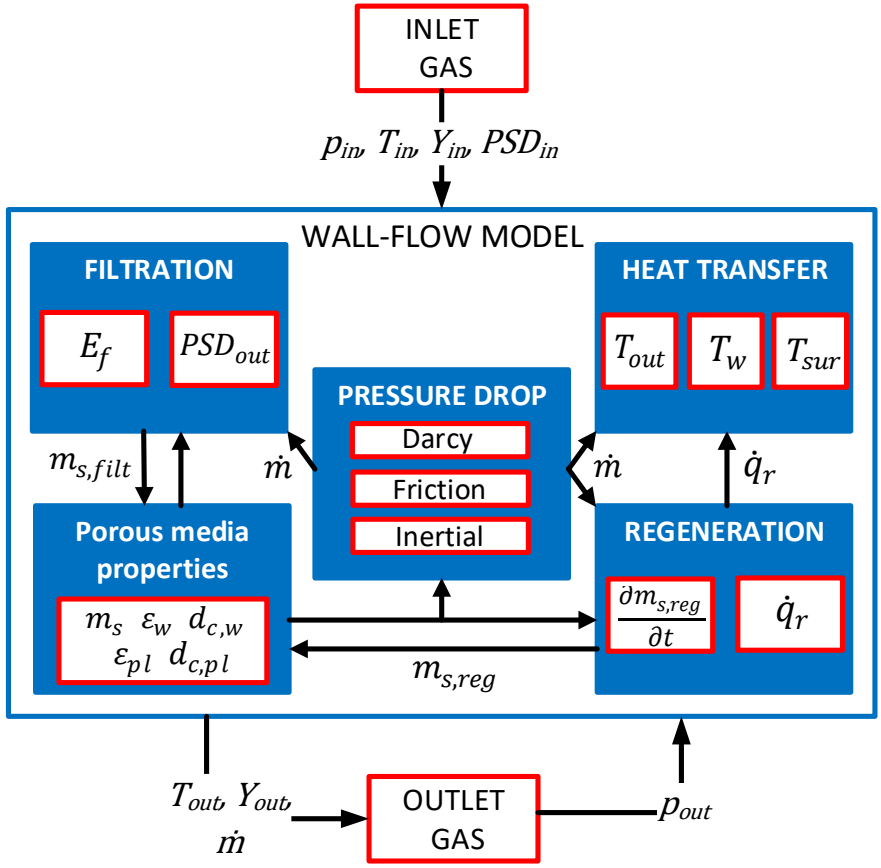

Figure 3. Flow-chart of wall-flow lumped model.

\section{Pressure drop}

The placement of the aftertreatment systems into the exhaust line involves a flow restriction directly related to the characteristics of their particular geometry and the flow properties. The pressure drop contributions are several. In flow-through monolithic reactors, these are mainly inertial contributions. The flow suffers a diffusion (pathline expansion) when enters the inlet volume as well as at the exit of the monolith channels. Complementarily, the flow is locally expanded (pathline contraction) at the channels inlet interface and when exiting from the outlet volume towards other monolith or the exhaust line. Assuming incompressible flow, the pressure drop in these kind of systems can be defined as

$$
\Delta p_{\text {cat }}=\frac{1}{2} \varsigma_{c a t} \rho_{\text {in }} u_{\text {in }}^{2}
$$

where $\varsigma_{c a t}$ is the pressure drop coefficient of the device. This coefficient is specific of the system geometry and a function of the Reynolds number. Taking the monolith inlet interface as reference for Reynolds number definition and characterizing experimentally the pressure drop coefficient value, the pressure drop can be imposed as boundary condition to obtain the flow velocity from Eq. (1). In turn, it allows calculating the mass flow across the catalyst every time-step from the inlet gas density and the open monolith cross-section.

In wall-flow monoliths, the importance of the inertial contributions is even higher due to the minor open area [26], with alternatively plugged channels and progressively reduced as soot is collected into the inlet channels. In addition to inertial and friction pressure drop, additional contributions appear due to the fact that the flow is forced to pass across the porous wall and the particulate layer, if this last exists because of soot or ash accumulation (Figure 4). 

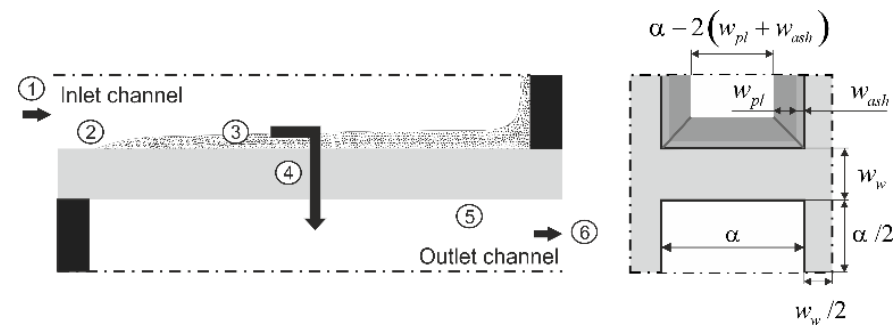

Figure 4. Pressure drop contributions into a wall-flow monolith: 1 - local flow expansion, 2 - inlet channel friction, 3 - particulate-ash layer, 4 - porous substrate, 5 - outlet channel friction, 6 - local flow diffusion.

Therefore, assuming incompressible flow, the particulate filter (PF) pressure drop can be expressed as [26]:

$$
\Delta p_{P F}=\Delta p_{\text {Darcy }}+\Delta p_{\text {inertial }}+\Delta p_{f r}
$$

The lumped approach requires neglecting the axial channel velocity parabolic profile [27]. Taking into account the cell geometry, which is shown in Figure 4, the representative channel velocity can be approximated as a function of the volumetric flow rate $(Q)$ and the inlet and outlet monolith cross-sections:

$$
u_{i n}=\frac{2 Q L_{e f}}{V_{e f} \sigma\left(\alpha-2\left(w_{p l}+w_{a s h}\right)\right)^{2}} ; u_{o u t}=\frac{2 Q L_{e f}}{V_{e f} \sigma \alpha^{2}}
$$

Therefore, as in flow-through monoliths, the inertial contribution to the pressure drop in a wall-flow PF is defined as

$$
\Delta p_{\text {inertial }}=\frac{1}{2} \varsigma_{t} \rho_{\text {in }} u_{\text {in }}^{2}
$$

which includes the total pressure drop coefficient $\left(\varsigma_{t}\right)$ related to the pressure drop due to flow diffusion (pathline expansion) at the inlet volume entrance, flow expansion (pathline contraction) at the monolith entrance, flow diffusion at the monolith outlet and flow expansion at the outlet volume. On the other hand, the friction contribution in square cross-section channels [28] is given by

$$
\Delta p_{f r}=\frac{1}{3} F_{w} \mu\left[\frac{u_{\text {in }} L_{\text {in }}}{\left(\alpha-2\left(w_{p l}+w_{\text {ash }}\right)\right)^{2}}+\frac{u_{\text {out }} L_{\text {out }}}{\alpha^{2}}\right]
$$

being $F_{w}$ the viscous loss coefficient set to 28.454 [10]. Finally, the pressure drop across the porous media is described by the Darcy's law [10] and by a minor Forchheimer's contribution, which can be assumed negligible in wall-flow monolith substrates [29].

The characteristic filtration velocity is dependent on the volumetric flow rate and the monolith geometry, i.e. the effective channel width for the flow transport $(\alpha(z))$, which varies across the particulate and ash layers from $\alpha-2\left(w_{p l}+w_{a s h}\right)$ to $\alpha$ as depicted in Figure 4:

$$
u_{w}=\frac{Q}{4 L_{\text {in }} \alpha(z) N_{\text {in }}}
$$

Making use of this definition, the porous medium pressure drop in every layer is given by

Page 4 of 22

$$
\Delta p_{\text {Darcy }}=\frac{\mu}{k} \int u_{w}(z) d z
$$

where $k$ is the permeability of the porous medium and $\mu$ the gas dynamic viscosity. Therefore, the total Darcy's contribution is obtained integrating Eq. (7) along the thickness of every porous medium. Therefore, the resulting Darcy's pressure drop can be calculated as:

$$
\Delta p_{\text {Darcy }}=\Delta p_{w}+\Delta p_{p l}+\Delta p_{a s h}
$$

Including the impact of the ash plug in the effective inlet channel length, it is finally obtained:

$$
\begin{gathered}
\Delta p_{\text {Darcy }}=\frac{\mu Q L_{e f}}{2 V_{e f} \sigma L_{\text {in }}}\left(\frac{w_{w}}{k_{w, e f} \alpha}+\frac{1}{2 k_{p l}} \ln \left(\frac{\alpha-2 w_{\text {ash }}}{\alpha-2\left(w_{p l}+w_{\text {ash }}\right)}\right)\right. \\
\left.+\frac{1}{2 k_{\text {ash }}} \ln \left(\frac{\alpha}{\alpha-2 w_{\text {ash }}}\right)\right)
\end{gathered}
$$

The use of this expression requires knowing different porous medium properties, some of them dependent on the soot load and the flow properties. The permeability of the clean porous wall is just dependent on the pore structure and the slip-flow correction [30]. As forward discussed, the clean porous wall permeability and the inertial pressure drop coefficient can be determined from the pressure drop measurement in a flow test rig. These data are the basis for the assessment of the permeability under loading conditions.

\section{Porous media properties}

The calculation of the pressure drop and the filtration efficiency in porous media is dependent on micro-geometry properties. The main parameters are the porosity and the mean pore diameter. Their definition in the porous wall, particulate layer and ash layer is based on the packed-bed of spherical particles theory, which considers the porous structure as a set of spherical unit cells. These unit cells fulfill that their porosity is the same as that of the porous medium being a unit collector placed in the core of the unit cell. In addition, the unit cells also have the same surface area to volume ratio as a pore with a diameter equal to the mean pore diameter.

In the porous substrate, the diameters of the unit collector $\left(d_{c, w}\right)$ and the unit cell $\left(d_{c e l l, w}\right)$ are functions of the porosity $\left(\varepsilon_{w}\right)$ and the mean pore diameter $\left(d_{p, w}\right)$. In clean conditions, these are respectively computed as:

$$
\begin{gathered}
d_{c, w_{0}}=\frac{3}{2}\left(\frac{1-\varepsilon_{w_{0}}}{\varepsilon_{w_{0}}}\right) d_{p, w_{0}} \\
d_{c e l l, w}=\sqrt[3]{\frac{d_{c, w_{0}}^{3}}{1-\varepsilon_{w_{0}}}}
\end{gathered}
$$

In turn, the permeability in clean conditions is also a function of the porosity and the mean pore diameter together with flow properties, which determine the slip-flow effect through the Stokes-Cunningham factor [31], 


$$
k_{w_{0}}=f\left(\varepsilon_{w_{0}}\right) d_{c, w_{0}}^{2} S C F_{w_{0}}
$$

where $f\left(\varepsilon_{w}\right)$ is a function of the Kuwabara's hydrodynamic factor (K) [30]

$$
\begin{aligned}
& f\left(\varepsilon_{w_{0}}\right)=\frac{0.02 K}{1-\varepsilon_{w_{0}}} \\
& =\frac{0.02\left(2-1.8\left(1-\varepsilon_{w_{0}}\right)^{\frac{1}{3}}-\varepsilon_{w_{0}}-0.2\left(1-\varepsilon_{w_{0}}\right)^{2}\right)}{1-\varepsilon_{w_{0}}}
\end{aligned}
$$

and the Stokes-Cunningham factor is dependent on the Knudsen number, which is defined as a function of the gas mean free path and the mean pore diameter. In a clean DPF, the SCF is obtained according to Eq. (14):

$$
\begin{gathered}
S C F_{w_{0}}=1+K n_{w_{0}}\left(1.257+0.4 e^{\frac{-1.1}{K n_{w_{0}}}}\right) \\
K n_{w_{0}}=\frac{2 \lambda}{d_{p, w_{0}}}
\end{gathered}
$$

All these properties can also be calculated analogously as a function of the soot load. As the soot is collected within the porous wall the variation of porosity and unit collector diameter is computed. The model applies a two layer porous wall concept due to the partial soot penetration $[1918,32,33]$. Therefore, the effective permeability is given by

$$
\Delta p_{w}=\frac{\mu u_{w} w_{w}}{k_{w, e f}}=\frac{\mu u_{w} w_{w} f_{s p}}{k_{w}}+\frac{\mu u_{w} w_{w}\left(1-f_{s p}\right)}{k_{w_{0}}}
$$

so that

$$
k_{w, e f}=\frac{k_{w} k_{w_{0}}}{f_{s p} k_{w_{0}}+\left(1-f_{s p}\right) k_{w}}
$$

where $f_{s p}$ is the fraction of porous wall thickness where the soot is collected.

The micro-geometric properties in this region are governed by the change in soot apparent density into the porous wall [19]. Assuming as soot packing density $\left(\rho_{s, w}\right)$ that of the soot aggregates with medium fractal dimension and medium number of primary particles $\left(345 \mathrm{~kg} / \mathrm{m}^{3}\right)$ [19], the apparent soot density is then a function of a shape factor related to the effective hydrodynamic diameter of the unit collector. It is identified by the dotted line in Figure 5(b).

a) Clean conditions

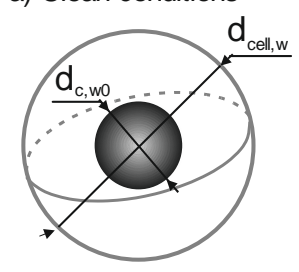

\section{b) Soot loading}

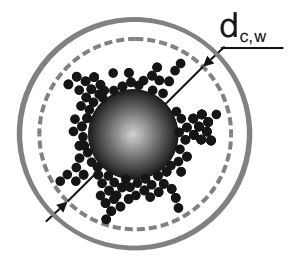

Therefore, the growth of the unit collector diameter can be expressed as

$$
d_{c, w}=2\left(\frac{d_{c, w_{0}}^{3}}{8}+\frac{3 m_{s, c e l l}}{4 \pi \chi \rho_{s, w}}\right)^{\frac{1}{3}}
$$

where the shape factor, which ranges between $0<\chi \leq 1$ and increases as the soot load within the porous wall does, is a function of the soot mass in the loaded porous wall fraction:

$$
\chi=a_{1}\left(\Phi_{\rho_{s}}\right)^{-a_{2}}+a_{3}
$$

In Eq. (19), $\Phi_{\rho_{s}}$ is the soot density factor, which is defined as the ratio between the soot packing density inside the porous wall and the soot mass to soot penetration volume ratio:

$$
\Phi_{\rho_{s}}=\frac{\rho_{s, w} V_{s p}}{m_{s, w}}
$$

Since the diameter of the unit collector varies as a function of the soot load within the unit cell, the porosity and the mean pore diameter are described by Eqs. (21) and (22) respectively:

$$
\begin{gathered}
\varepsilon_{w}=1-\left(\frac{d_{c, w}}{d_{c e l l, w}}\right)^{3} \\
d_{p, w}=\frac{2}{3}\left(\frac{\varepsilon_{w}}{1-\varepsilon_{w}}\right) d_{c, w}
\end{gathered}
$$

The parameter governing the switch from deep bed to cake filtration regime is the saturation coefficient provided that there is not an ash layer. If it is already formed, it will act as a barrier filter imposing cake filtration from the very beginning of the loading process. The saturation coefficient relates the amount of collected soot to the maximum amount that can be collected per unit cell in volumetric terms as

$$
F_{s a t}=\frac{d_{c, w}^{3}-d_{c, w_{0}}^{3}}{\left(\varphi d_{c e l l, w}\right)^{3}-d_{c, w_{0}}^{3}}
$$

where $\varphi$ represents the percolation factor. The saturation mass of the unit cell is proportional to the difference between the cell and the clean unit collector volumes according to Eq. (24):

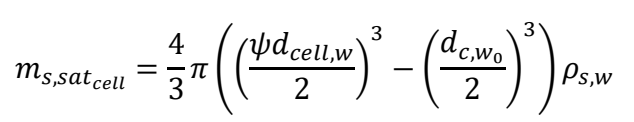

The permeability in the particulate and ash layers is calculated applying Eq. (12) adapted to these substrates. In the case of the particulate layer, the unit collector diameter is set to the mode of the particulate size distribution, which makes the porosity to fall typically into the range 0.6 to 0.7 [19]. This range is in agreement with the porosity of the mean soot aggregates $(\sim 0.81)[34,35]$ and the ballistic deposition, i.e. governed by convective transport, of the soot onto the porous wall, which leads to particulate layer compaction [36,37].

$$
k_{p l}=f\left(\varepsilon_{p l}\right) d_{c, p l}^{2} S C F_{p l}
$$




$$
k_{a s h}=f\left(\varepsilon_{a s h}\right) d_{c, a s h}^{2} S C F_{a s h}
$$

In these layers, the Stokes-Cunningham factor is calculated from the Knudsen number referred to the gas mean free path and the mean pore diameter of every layer. It is obtained according to Eq. (10), which is referred to the clean porous wall. The porosity of the layer and the unit collector diameter, which is defined as the characteristic size of the deposited particles, are used.

\section{Filtration}

The filtration sub-model computes the mass-based filtration efficiency and, thus, the amount of soot collected per unit cell. The model provides both the overall filtration efficiency, which is represented by that corresponding to the mode diameter of the particle emissions, and as a function of the particle size distribution (PSD) [18]. It is done accounting by Brownian and interception collection mechanisms around a single sphere. The inertial contribution is not included due to its negligible impact [18].

Brownian diffusion that aerosol particles undergo gets importance as the particle size decreases. In addition, Brownian diffusion is also favoured when the flow velocity is small, so that it dominates the particle motion. It happens in wall-flow particulate filters, which are characterised by high filtration area and, hence, low filtration velocity. Consequently, the small particles are able to leave the streamlines diffusing away towards the unit collectors on which they are finally deposited. The single collector efficiency for Brownian diffusion is given by [38]

$$
\eta_{D}=3.5\left(\frac{\varepsilon_{w}}{K}\right)^{\frac{1}{3}} P e_{w}^{-\frac{2}{3}}
$$

where the Peclet number $\left(P e_{w}\right)$ is defined as:

$$
P e_{w}=\frac{u_{w} d_{c, w}}{\varepsilon_{w} D_{\text {part }}}
$$

As shown in Eq. (28), the Peclet number is a function of the flow and the loaded porous wall properties. The soot load contributes to increase the particles collection. It is the main governing parameter of the collection process and leads to high filtration efficiency. Nevertheless, the flow properties have important effects when the soot loading is low. Its influence is mainly related to the mass flow and the gas temperature. The gas temperature directly affects the particle diffusion coefficient according to Eq. (29). In addition, the gas density is also affected leading to a relevant influence on the filtration velocity for a given mass flow. The gas density is also dependent on the gas pressure, but this last has minor importance in standard post-turbine PF location where its value is closer to ambient conditions. Nevertheless, it can also get importance in pre-turbine PF configurations, thus affecting the filtration velocity and the slip-flow effect [39].

$$
D_{\text {part }}=\frac{T k_{B} S C F_{w}}{3 \pi \mu d_{\text {part }}}
$$

In Eq. (29), $k_{B}$ is the Boltzmann constant and $d_{\text {part }}$ is the particle diameter, which has an opposite effect to that of the gas temperature.
The interception efficiency of a single unit collector is related to the inability of the particles to deviate from the streamline being stuck on the unit collector if it brings the particle surface within the radius of the collector surface. The contribution of this mechanism, which takes importance as the particle size increases, i.e. inversely to Brownian diffusion, is quantified according to Eq. (30)

$$
\eta_{R}=1.5 \frac{N_{R}^{2}}{1+N_{R}^{\frac{3-2 \varepsilon_{w}}{3 \varepsilon_{w}}}} \frac{\varepsilon_{w}}{K}
$$

where $N_{R}$ is the interception parameter defined as the ratio between the particle size and unit collector diameter.

Finally, making use of the independence rule to consider the mutual effect of both mechanisms, the overall filtration efficiency of an isolated single collector is computed as:

$$
\eta_{D R}=\eta_{D}+\eta_{R}-\eta_{D} \eta_{R}
$$

From the collection efficiency of a single unit collector, the filtration efficiency of the packed spherical particles representing the porous wall is given by:

$$
E_{f}=1-e^{-\frac{3 \eta_{D R}\left(1-\varepsilon_{w}\right) w_{w} f_{s p} S_{c}}{2 \varepsilon_{w} d_{c, w}}}
$$

Eq. (32) is obtained from the mass balance of particles through the packed bed control volume using the pore velocity as characteristic velocity for the particles due to the proximity among collectors [40]. The prediction of the filtration efficiency in clean conditions is depending on the sticking coefficient $\left(S_{c}\right)$, which accounts for the ratio between the rate of particles stick to the collector and the rate they strike it [40]. It is a parameter obtained empirically and used to correct the collection efficiency of the single sphere [40]. The filtration efficiency in clean conditions is very sensitive to this parameter. However, its influence is minor on the filtration efficiency variation as a function of the soot loading and on its maximum value [18]. As the soot load increases, the dynamic growth of the filtration efficiency is governed by the change in porous wall micro-geometry as well as on the soot penetration. This parameter has also a direct impact on the pressure drop prediction. The modelling of loading processes in different DPFs revealed a linear dependence between the soot penetration thickness and the Peclet number in the porous wall at the very beginning of the loading process [18] according to Eq. (33):

$$
w_{s p}=w_{w} f_{s p}=0.0019 P e_{w_{0}}
$$

Although the effects of transition from deep bed to cake filtration regimes are mainly related to the one-dimensional flow distribution [18], i.e. the filtration velocity profile along inlet channels, the porous wall properties play also a role. Despite the fact that it is not very relevant in quantitative terms, a transition phase modelling avoids discontinuities over time in filter performance prediction, such as pressure drop and filtration efficiency [18]. Therefore, two mechanisms are considered to model the transition from deep bed to cake filtration regime. On the one hand, a limit saturation coefficient $\left(S_{l}\right)$ is defined. When the saturation coefficient of the unit cell exceeds this value, the particulate layer starts to grow governed by the particulate layer filtration efficiency. The growth is assumed to be a linear function of the porous wall filtration efficiency. The particulate layer filtration efficiency converges to that of the porous wall once this is saturated [18]. It is because the porous wall acts as a 
barrier as it is saturated so that incoming particles are deposited on its surface increasing the thickness of the particulate layer. Therefore, the particulate layer filtration efficiency can be expressed as [18]:

$$
E_{f, p l}=E_{f, w}\left(\frac{F_{s a t}-S_{l}}{1-S_{l}}\right)
$$

In parallel to this mechanism, a hill-like local growth of the particulate layer around pores is proposed based on the experimental findings of Choi and Lee [41]. A homogenous distribution of soot on the filtration area (porous wall surface of the inlet channels) allows calculating the particulate layer thickness as a function of the soot mass in the particulate layer, the particulate layer density and the occupied volume. Taking into account the hill-like local growth around the pores, the resulting particulate layer thickness can be corrected to increase while the thickness is below a threshold value. This approach is equivalent to consider that the soot mass is concentrated around the surface pores leading to higher local particulate layer thickness, as schematically represented in Figure 6. Therefore, the particulate layer thickness can be expressed as

$$
w_{p l}=\frac{w_{p l_{\text {hom }}}}{\xi}=\frac{\alpha-\sqrt{\alpha^{2}-\frac{m_{s, p l}}{L_{i n} \rho_{p l}}}}{2 \xi}
$$

where $\xi$ is the correction factor. According to [18], it is proposed a correction factor varying linearly from the porous wall porosity when the particulate layer starts to grow up to one once the limit particulate layer thickness is reached. Therefore, it is obtained as:

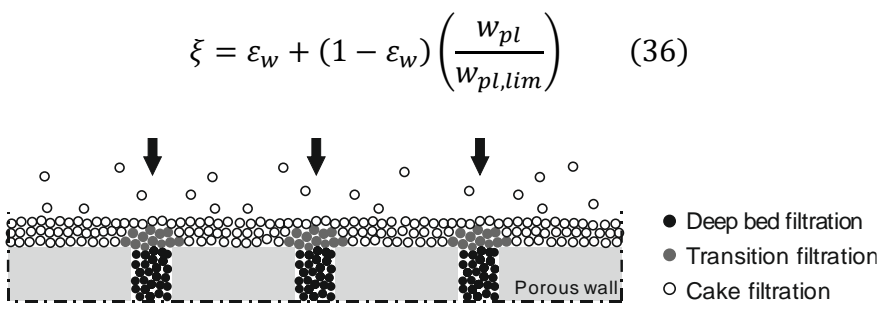

Figure 6. Particulate layer growth during the transition phase: soot deposition onto the porous wall around the border region of surface pores.

\section{Reaction mechanism}

The chemical conversion within the monolith is tackled integrating the one-dimensional chemical transport equations. It is considered in the axial channel direction for gaseous emissions in flow-through monoliths and across the porous wall to model the soot oxidation in wall-flow monoliths.

\section{Gaseous emission modelling}

The chemical reaction sub-model for gaseous pollutant emissions computes their conversion efficiency integrating the one-dimensional chemical species transport equation into the gas stream and the washcoat along the monolith length [42]. Assuming quasi-steady flow, these governing equations are given by

$$
\begin{gathered}
u_{i n} \frac{d X_{n}}{d x}=-S_{p_{c a t}} k_{m, n}\left(X_{n}-X_{n, S}\right) \\
v_{n} R_{n}+S_{p_{w c}} k_{m, n}\left(X_{n}-X_{n, S}\right)=0
\end{gathered}
$$

where Eq. (37) regards the bulk gas equation. The left-hand side accounts for the convection transport of the species along the channel and the right-hand side represents the diffusion of species from the bulk to the channel surface. Complementarily, Eq. (38) represents the chemical species transport over the catalyst surface. It comprises the diffusion of the species from/to the surface and to/from the washcoat and the corresponding reaction rates. $X_{n}$ and $X_{n, S}$ are the molar fraction of species $n$ into the gas and in the washcoat respectively, $S_{p_{\text {cat }}}$ represents the geometric surface area related to the mass transfer from the gas to the catalyst surface and $S_{p_{w c}}$ is the geometric surface area related to the mass transfer from the catalyst surface into the washcoat volume. These geometric surface areas are defined as

$$
\begin{gathered}
S_{p_{c a t}}=\frac{S_{\text {cat }}}{V_{g a s}}=\frac{S_{p_{\text {mon }}}}{O F A} \\
S_{p_{w c}}=\frac{S_{\text {cat }}}{V_{w c}}=\frac{S_{p_{\text {mon }}}}{(1-O F A) \Theta_{w c}}
\end{gathered}
$$

where $O F A$ is the open frontal area of the monolith, $\Theta_{w c}$ represents the washcoat fraction over the solid area and $S_{p_{m o n}}$ is the geometric surface area of the monolith $\left(S_{\text {cat }} / V_{\text {mon }}\right)$. Expressing these variables as a function of the cellular geometry parameters, i.e. channel width and wall thickness, Eqs. (39) and (40) can be adapted to diverse monolithic channel cross sections.

On the other hand, the external diffusion from bulk gas to catalyst external surface is represented by the bulk mass transfer coefficient $k_{m}$. It is defined as a function of the Sherwood number and the molecular diffusivity for every pollutant species as

$$
k_{m, n}=\frac{D_{m_{n}} S h_{n}}{\alpha}
$$

being the Sherwood number $(S h)$ defined as proposed by Hawthorn [43] for square channel cross-section and the molecular diffusivity obtained from the mixture of every species $n$ with the exhaust gas compounds (see Eq.(48)). Finally, the reaction rate for every pollutant species $n$ accounts for all reactions in which is involved. In a general case, the reaction rate in gas phase reactions is expressed as

$$
R_{r, n}=\eta_{i n t, r, n} \frac{k_{r, n}}{G_{r, n}}\left(\prod_{j} X_{j}^{m_{j}}-\frac{\prod_{i} X_{i}^{m_{i}}}{K_{e q, r, n}}\right)
$$

where $j$ denotes the reactants in the forward reactions, $i$ the products (reactants in the reverse reaction) and $m$ is the reaction order. Eq. (42) is a general representation of the reaction rate that is valid to solve forward and reverse reactions [44]. From the calibration of the kinetic constant in the forward reaction, the use of the equilibrium constant $\left(K_{e q, r, n}\right)$ allows calculating the reaction rate of the driven reverse reaction. In adsorption processes leading to gas compounds accumulation and the subsequent desorption, the reaction rate of the species $n$ is written as shown next:

$$
\begin{gathered}
R_{a d s, n}=\eta_{i n t, a d s, n} \frac{k_{a d s, n}}{G_{a d s, n}}(1-\theta) \psi \prod_{j} X_{j}^{m_{j}} \\
R_{\text {des }, n}=\eta_{\text {int }, \text { des }, n} \frac{k_{\text {des }, n}}{G_{\text {des }, n}} \theta_{n} \psi \prod_{j} X_{j}^{m_{j}}
\end{gathered}
$$


In Eqs. (43) and (44) $\theta$ represents the surface coverage and $\psi$ is the specific storage capacity of the substrate.

In every reaction, the kinetic constant $k_{r, n}$ is expressed by means of an Arrhenius type equation dependent on the inlet gas temperature; $G_{r, n}$ is the inhibition term [45] and $\eta_{i n t, r, n}$ represents the internal pore diffusion efficiency. The calculation of the internal pore diffusion efficiency is performed assuming isothermal conditions. It is because the rate of change of the substrate temperature is very low and can be considered negligible within every calculation time-step, as discussed in next sections. Therefore, the internal pore diffusion efficiency can be computed as a function of the Thiele modulus [46]:

$$
\eta_{i n t, r, n}=\frac{1}{\phi_{r, n}}\left(\frac{1}{\tanh \left(3 \phi_{r, n}\right)}-\frac{1}{3 \phi_{r, n}}\right)
$$

Since the diffusion process in the meso-pores of the catalyst particle is more limiting than in the macro-pores of the catalyst slab [47], the Thiele modulus is defined assuming spherical catalyst particles [48] as $[49,50]$

$$
\phi_{r, n}=\frac{r_{c a t}}{3} \sqrt{\frac{k_{r, n} \prod_{j} X_{j}^{m_{j}}}{D_{e f, n} X_{n}}}
$$

where $D_{e f, n}$ is the effective diffusivity of the species $n$. It is calculated as proposed by Edelbauer et al. [51]

$$
D_{e f, n}=\frac{\varepsilon_{c a t}}{\omega}\left(\frac{1}{D_{m_{n}}}+\frac{1}{D_{K n_{n}}}\right)^{-1}
$$

where the molecular diffusivity in the mixture of gases is obtained as [52]

$$
D_{m_{n, l}}=\frac{1.43 \times 10^{-6} \frac{T^{1.75}}{p}}{\sqrt{\frac{M_{n}+M_{l}}{0.002}}\left(v_{n}^{\frac{1}{3}}+v_{l}^{\frac{1}{3}}\right)^{2}} \rightarrow D_{m_{n}}=\left(\sum_{k} \frac{X_{k}}{D_{m_{n, l}}}\right)^{-1}
$$

and the Knudsen diffusivity according to [46]

$$
D_{K n_{n}}=\frac{d_{p, c a t}}{3} \sqrt{\frac{8 \Re T}{\pi M_{n}}}
$$

where $d_{p, c a t}$ is the pore diameter of the catalyst particle.

The solution of the chemical species conservation equations provides the rate of variation of the pollutant emissions. Therefore, applying the stoichiometry of the reactions defining the particular reaction mechanism to be applied in every monolithic reactor, the released heat per unit of time onto the substrate can be represented in a general way as:

$\dot{q}_{r}=\dot{n}_{T, g a s} \sum_{j} H_{f, j} \Delta X_{j}+\sum_{a-s} \Psi_{T, a-s} \frac{\sum_{i} \Delta H_{\frac{a d s}{d e s}, a-s, i} \Delta \theta_{a-s, i}}{\Delta t}$ catalyst, $H_{f, j}$ is the enthalpy of formation of the species $j$ and $\Delta X_{j}$ its molar fraction variation during the time-step due to the gas phase reaction mechanism. The second summation accounts for the heat of reaction related to adsorption and desorption processes in the catalyst active sites. In this summation $\Psi_{T, S}$ is the maximum amount of moles

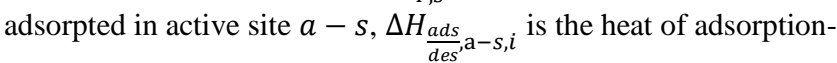
desorption of species $i$ in active site $a-s$ and $\Delta \theta_{a-s, i}$ is the coverage variation for species $i$.

\section{Soot oxidation modelling}

The regeneration sub-model in wall-flow particulate filters solves the conservation equation of the soot oxidizing species accounting for a three-layer step. As sketched in Figure 7, particulate layer, coated porous wall and uncoated porous wall region are distinguished.

The model considers that the soot oxidation in every region takes places in presence of $\mathrm{O}_{2}$, which becomes predominant at high temperatures and in catalysed filters, and $\mathrm{NO}_{2}$, which is the main oxidant at low temperature [53].

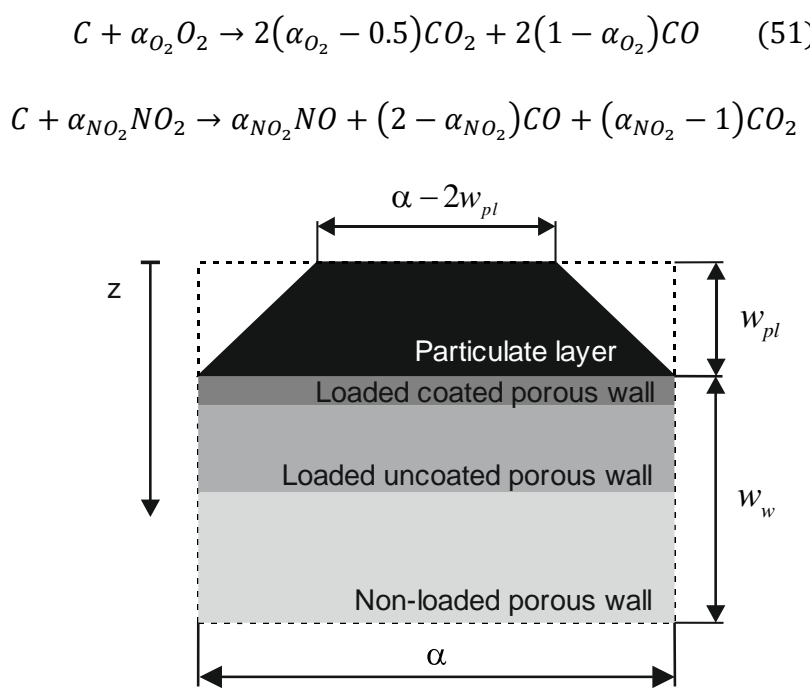

Figure 7. Scheme of the regions of soot regeneration in the wall-flow particulate filter.

Assuming quasi-steady flow and including the influence of external and internal diffusion, the transport of $\mathrm{O}_{2}$ and $\mathrm{NO}_{2}$ in the tangential direction of every layer is given by

$$
u_{w} \frac{d X_{n}}{d z}-D_{m_{n}} \frac{d^{2} X_{n}}{d z^{2}}=-S_{p_{s}} k_{r e g, n} \alpha_{n} \theta_{n} \eta_{i n t, n}
$$

where the reaction rate is proportional to the Arrhenius type kinetic constant $k_{n}$, which is defined as a function of the inlet gas temperature, the soot geometric surface area $\left(S_{p_{s}}\right)$ [9] and the internal pore diffusion efficiency of species $n$ related to the soot particle properties $[54,55]$. The reaction order is depending on the operating conditions as a function of the surface coverage $\theta_{n}$, i.e. the fraction of surface positions in the soot particle that are occupied by the reactant species. This adsorption step is modelled by means of the Langmuir isotherm $[56,57]$ as
In Eq. (50), the first summation refers to gas phase reactions, where $\dot{n}_{T, \text { gas }}$ represents the total exhaust gas mole flow entering the Page 8 of 22 


$$
\theta_{n}=\frac{K_{e q, \mathrm{~s}, n} X_{n}}{1+K_{e q, \mathrm{~s}, n} X_{n}}
$$

where $K_{e q, s, n}$ is the equilibrium constant for the adsorption and desorption processes of the oxidizing agent on the soot surface. Thus, Eq. (53) is solved for $\mathrm{O}_{2}$ and $\mathrm{NO}_{2}$ applying a $4^{\text {th }}$-order Runge-Kutta method consecutively to every layer. It means that the solution in every layer is decoupled. The boundary conditions are imposed at the inlet of every layer. They are the concentration at the outlet of the previous region (inlet gas concentration in the first layer) and the first derivative of the concentration in the tangential direction. This last is calculated as a function of the reaction rate and the diffusion term, which is approximated at the beginning of the time-step. Therefore, an iterative procedure is applied until the convergence is obtained.

Known the $\mathrm{O}_{2}$ and $\mathrm{NO}_{2}$ depletion rate at every layer from its molar fraction variation, the filtration velocity, the filtration area and the molar concentration, i.e.

$$
\frac{\partial n_{n}}{\partial t}=\Delta X_{n} u_{w} A_{f} c_{g a s}
$$

the soot depletion rate is then obtained accounting for the completeness index of every reaction as:

$$
\frac{\partial m_{s, r e g}}{\partial t}=-M_{c} \sum_{n} \frac{1}{\alpha_{n}} \frac{\partial n_{n}}{\partial t}
$$

Finally, the released heat per unit of time during the regeneration process on the porous media is calculated accounting for the stoichiometry coefficients and the enthalpy of formation of the involved species according to Eq. (50).

\section{Heat transfer}

The heat transfer sub-model applies a lumped nodal approach based on the discretization of the general heat transfer equation:

$$
\rho c_{p} \frac{\partial T}{\partial t}=\left(\frac{\partial^{2} T}{\partial x^{2}}+\frac{\partial^{2} T}{\partial z^{2}}\right) \kappa+\dot{q}_{r}^{\prime \prime}
$$

Discretizing this equation by centered finite differences, the wall temperature at time-step $p+1$ and node $(i, j)$ can be solved explicitly from the wall temperature at time-step $p$ :

$$
\begin{gathered}
\rho c_{p} \frac{T_{i, j}^{p+1}-T_{i, j}^{p}}{\Delta t}=\kappa\left(\frac{T_{i-1, j}^{p}-2 T_{i, j}^{p}+T_{i+1, j}^{p}}{(\Delta x)^{2}}\right. \\
\left.+\frac{T_{i, j-1}^{p}-2 T_{i, j}^{p}+T_{i, j+1}^{p}}{(\Delta y)^{2}}\right)+\dot{q}_{r}^{\prime \prime}
\end{gathered}
$$

Taking into account the characteristics of every control volume and different thermal properties and heat transfer area at every node, the wall temperature can be finally calculated as:

$T_{i, j}^{p+1}=\frac{\Delta t}{C_{i, j}}\left(\sum_{k=-1}^{+1} \frac{\mathrm{T}_{i+k, j}^{p}-T_{i, j}^{p}}{R_{i+k, j} / i, j}+\sum_{k=-1}^{+1} \frac{\mathrm{T}_{i, j+k}^{p}-T_{i, j}^{p}}{R_{i, j+k / i, j}}+\dot{q}_{r}\right)+T_{i, j}^{p}$

In Eq. (59), $C_{i, j}$ represents the thermal capacity in the control volume of node $(i, j) ; R$ is the equivalent thermal resistance between two neighboring nodes.

Page 9 of 22
The use of simple lumped heat transfer models has gained an increasing attention due to their reasonable compromise between computational cost and solution accuracy. As shown in Eq. (59), this kind of approach can provide an explicit solution of the wall temperature in complex systems, such as the engine [58] or the turbocharger [59]. In exhaust aftertreatment systems, where the exhaust gas exchanges heat with the environment via the substrate material and the external canning, these models also find a field of application. The definition of a representative network of equivalent thermal resistances and capacitances to account for heat exchange and thermal inertia of the monolith substrate and the external canning is required. Figure 8 shows the proposed nodal scheme in this work. The lumped representation of the monolith has been adapted from the 1D heat transfer model proposed by Galindo et al. [17] for wall-flow PFs.

a) Monolith nodal scheme

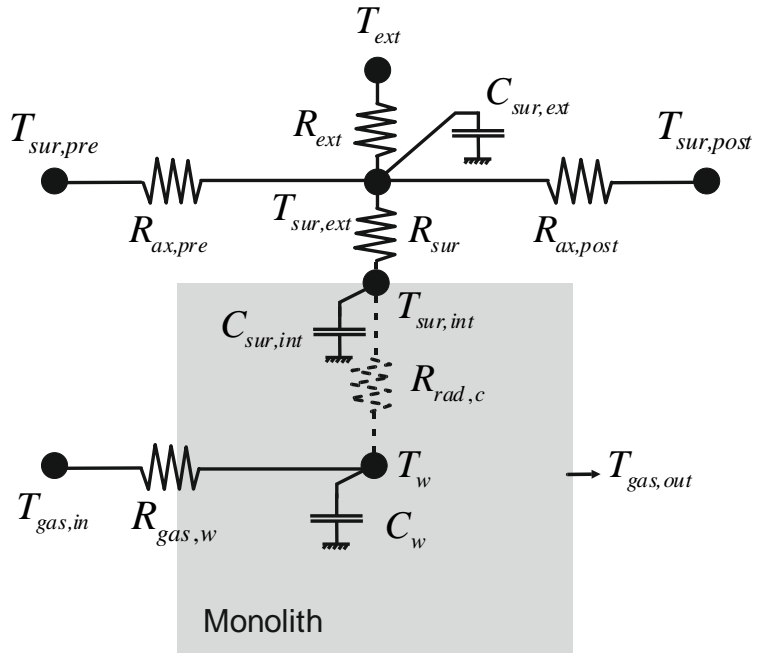

b) Surface equivalent thermal resistance

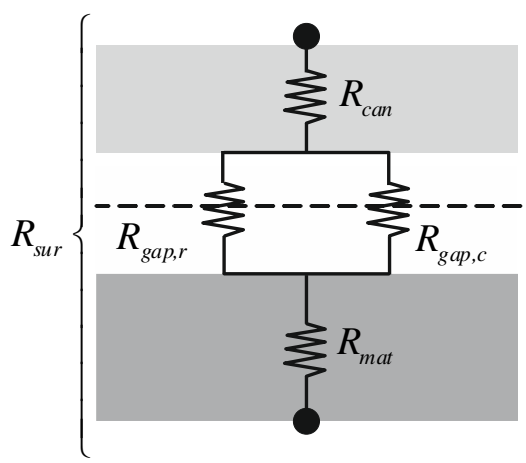

Figure 8. Nodal scheme of the heat transfer sub-model.

The heat transfer in flow-through and wall-flow monoliths is modelled in the same way just taking into account the particular geometry characteristics related to gas to wall heat transfer.

According to Figure 8, the model solves the heat transfer equation in the nodes located on the substrate and the external canning. The substrate wall temperature $\left(T_{w}\right)$ is estimated with the objective to provide the outlet gas temperature. The lumped assumption involves that only averaged monolith conditions are calculated. Consequently, the lack of axial resolution avoids capturing local effects with the proposed sub-model. However, radial discretization is possible. It allows describing the averaged wall temperature gradient towards the 
monolith periphery, where the internal surface temperature $\left(T_{\text {sur,int }}\right)$ is assessed. The heat transfer across the canning is modelled from this boundary node accounting for the different layers to predict the external surface temperature $\left(T_{\text {sur,ext }}\right)$, which is also dependent on the surrounding elements and the ambient temperature.

Based on the definition of the thermal equivalent resistances and capacitances shown in Figure 8 and detailed in the Appendix, and adding the heat released due to chemical reactions, the substrate wall temperature can be obtained as

$$
T_{w}^{B, p+1}=\frac{\Delta t}{C_{w}^{B, p}}\left(\sum_{k} \frac{T_{k}-T_{w}^{B, p}}{R_{k}}+\dot{q}_{r}^{p}\right)+T_{w}^{B, p}
$$

where superscripts $B$ and $p$ represent the channels beam and the timestep to which every variable belongs. The summation considers all the thermal equivalent resistances connected to the substrate wall node, i.e. the one representing gas to wall heat transfer in the monolith channels and those related to radial conduction from the monolith core to the external surface.

The internal and external canning temperatures are computed in the same way, thus obtaining:

$$
\begin{gathered}
T_{\text {sur }, \text { int }}^{p+1}=\frac{\Delta t}{C_{\text {sur }, \text { int }}^{p}}\left(\frac{T_{w}^{B_{\text {per }, p}}-T_{\text {sur }, \text { int }}^{p}}{R_{\text {rad }}^{B_{\text {per }}, p}}+\frac{T_{\text {sur }, \text { ext }}^{p}-T_{\text {sur }, \text { int }}^{p}}{R_{\text {sur }}^{p}}\right) \\
+T_{\text {sur }, \text { int }}^{p} \\
T_{\text {sur }, \text { ext }}^{p+1}=\frac{\Delta t}{C_{\text {sur }, \text { ext }}^{p}}\left(\frac{T_{\text {ext }}-T_{\text {sur }, \text { ext }}^{p}}{R_{\text {ext }}^{p}}+\frac{T_{\text {sur }, \text { int }}^{p}-T_{\text {sur }, \text { ext }}^{p}}{R_{\text {sur }}^{p}}\right. \\
\left.+\frac{T_{\text {sur }, \text { pre }}^{p}-T_{\text {sur }, \text { ext }}^{p}}{R_{\text {ax }, \text { pre }}^{p}}+\frac{T_{\text {sur }, \text { ost }}^{p}-T_{\text {sur }, \text { ext }}^{p}}{R_{\text {ax }, \text { post }}^{p}}\right)+T_{\text {sur }, \text { ext }}^{p}
\end{gathered}
$$

\section{Outlet gas properties}

The outlet gas properties are obtained as the last stage of the solution process from the results provided by the pressure drop, heat transfer and chemical conversion sub-models previously described. Thus, the energy and mass balance between inlet and outlet sections of the monolith are solved every time-step yielding the outlet gas temperature and velocity:

$$
\begin{gathered}
T_{\text {out }}=\frac{c_{p_{\text {in }}}}{c_{p_{\text {out }}}} T_{\text {in }}-\frac{\dot{q}_{\text {ht }}}{\dot{m} c_{p_{\text {out }}}}+\frac{u_{\text {in }}^{2}-u_{\text {out }}^{2}}{2 c_{p_{\text {out }}}} \\
u_{\text {out }}=\frac{A_{\text {in }} u_{\text {in }} p_{\text {in }} T_{\text {out }}}{A_{\text {out }} p_{\text {out }} T_{\text {in }}}
\end{gathered}
$$

On the other hand, the outlet gas composition of every species $n$ can be expressed as a function of the inlet mass fraction and the variation in mass fraction of reactants and products. In the general case, i.e. including particulate matter filtration, the outlet mass fraction of chemical species $n$ is given by

$$
Y_{n, \text { out }}=\frac{\dot{m}_{n, \text { out }}}{\dot{m}_{\text {out }}}=\frac{\dot{m}_{n, \text { in }}+\dot{m}_{\text {in }} \Delta Y_{n}}{\dot{m}_{\text {in }}\left(1+\sum \Delta Y_{i}\right)}=\frac{Y_{n, \text { in }}+\Delta Y_{n}}{1+\sum \Delta Y_{i}}
$$

where the mass fraction variation is determined from filtration efficiency for soot and from the solution of Eqs. (37) and (38) for gaseous pollutants. The mass fraction variation of the non-pollutant chemical species is calculated from the stoichiometry of every chemical reaction:

$$
\Delta Y_{n}=-\frac{M_{n}}{\bar{M}} \sum_{j} v_{n} \Delta X_{j}
$$

In eq. (66) $M_{n}$ and $\bar{M}$ are the molecular weights of the species $n$ and the gas mixture respectively, $v_{n}$ is the stoichiometric coefficient of the species $n$ ( $<0$ for reactants, $>0$ for products) and $\Delta X_{j}$ represents the molar fraction variation of the pollutants involving species $n$ in their conversion.

\section{Experimental validation}

The modelling of a close-coupled DOC and DPF brick was carried out as validation. Several in-engine experiments were selected to characterize specific phenomena under well-controlled conditions. The last step was the modelling of a Worldwide harmonized Light vehicles Test Cycle (WLTC), in which all processes takes place simultaneously. This test was also used to quantify the computational effort of the DOC and DPF models in order to assess their potential for real-time applications. The tests were performed with a Euro 5 passenger car Diesel engine, whose main characteristics are summarized in Table 1. The geometrical parameters of the aftertreatment systems are also listed in Table 2.

Table 1. Main engine characteristics.

\begin{tabular}{|l|l|}
\hline Type & Euro 5 HSDI Diesel engine \\
\hline Displaced volume & $1598 \mathrm{cc}$ \\
\hline Stroke & $79.5 \mathrm{~mm}$ \\
\hline Bore & $80 \mathrm{~mm}$ \\
\hline Number of cylinders & 4 in line \\
\hline Number of Valves & 4 per cylinder \\
\hline Compression ratio & $14.5: 1$ \\
\hline Turbocharging & VGT \\
\hline EGR system & SR-EGR, LR-EGR \\
\hline Maximum power @ speed & $96 \mathrm{~kW} @ 4000 \mathrm{rpm}$ \\
\hline Maximum torque @ speed & 320 Nm @ 1750 rpm \\
\hline
\end{tabular}

Table 2. Geometric characteristics of DOC and DPF.

\begin{tabular}{|l|l|l|l|}
\hline \multicolumn{2}{|c|}{ DOC } & \multicolumn{2}{c|}{ DPF } \\
\hline Diameter & $0.172 \mathrm{~m}$ & Diameter & $0.172 \mathrm{~m}$ \\
\hline Length & $0.082 \mathrm{~m}$ & Length & $0.1 \mathrm{~m}$ \\
\hline Cell density & $400 \mathrm{cpsi}$ & Plug length & $5 \mathrm{~mm}$ \\
\hline Channel width & $1.17 \mathrm{~mm}$ & Cell density & $200 \mathrm{cpsi}$ \\
\hline Wall thickness & $0.101 \mathrm{~mm}$ & Channel width & $1.39 \mathrm{~mm}$ \\
\hline $\mathrm{N}^{\text {o }}$ of channels & 14400 & Wall thickness & $0.4 \mathrm{~mm}$ \\
\hline Catalytic area & $5.5 \mathrm{~m}^{2}$ & $\mathrm{~N}^{\mathbf{o}}$ of channels & 7200 \\
\hline $\begin{array}{l}\text { Geometric } \\
\text { surface area }\end{array}$ & $2900 \mathrm{~m}^{-1}$ & Filtration area & $2.11 \mathrm{~m}{ }^{2}$ \\
\hline Channel section & Square & Channel section & Square \\
\hline
\end{tabular}




\section{DOC modelling}

As previously discussed, the mass flow across the monolith cannot be imposed in gas dynamics applications. It is predicted from inlet and outlet pressure, which set problem boundaries. Therefore, the pressure drop coefficient of the DOC was firstly determined as a function of the Reynolds number. It was done making use of motoring tests. These tests are useful for this purpose since only pressure drop takes place. Heat transfer can be assumed negligible because of the low operating temperature, which is very close to ambient conditions, whilst chemical reactions are completely avoided because of the lack of reacting flow.

Figure 9 shows the prediction of mass flow across the DOC when the experimental pressure drop is imposed. The steps in Figure 9(a) correspond to different engine speeds under motoring operation. The experimental instantaneous pressure drop coefficient was calculated according to Eq. (1) for every operating point. The average value of every point, which are black circled in Figure 9(b), was used to obtain a fitting function covering a wider Reynolds number range to be applied to predict the mass flow under different operating conditions.

Once the DOC pressure drop behavior was characterized, a set of steady-state tests were run as a basis for the heat transfer and chemical sub-models setup. As a particular case of flow-through monolith, the main chemical functions of the DOC comprise the oxidation of $\mathrm{CO}$ and $\mathrm{HC}$. In addition, the DOC also affects the $\mathrm{NO}_{2}$ to $\mathrm{NO}$ ratio being the objective to move this ratio towards the equilibrium conditions. It makes the $\mathrm{NO}_{2}$ concentration increase and benefits the DPF passive regeneration and the NOx abatement in SCR devices. Therefore, the lumped model computes the variations in gas composition taking into account the following reactions:

$$
\begin{aligned}
& \mathrm{CO}+\frac{1}{2} \mathrm{O}_{2} \rightarrow \mathrm{CO}_{2} \\
& \mathrm{C}_{n} \mathrm{H}_{m}+\left(n+\frac{m}{4}\right) \mathrm{O}_{2} \rightarrow n \mathrm{CO}_{2}+\frac{m}{2} \mathrm{H}_{2} \mathrm{O} \\
& \mathrm{C}_{n} \mathrm{H}_{m}+\text { Zeolite } \leftrightarrows \mathrm{C}_{n} \mathrm{H}_{m} \cdot \text { Zeolite } \\
& \mathrm{NO}+\frac{1}{2} \mathrm{O}_{2} \leftrightarrows \mathrm{NO}_{2}
\end{aligned}
$$

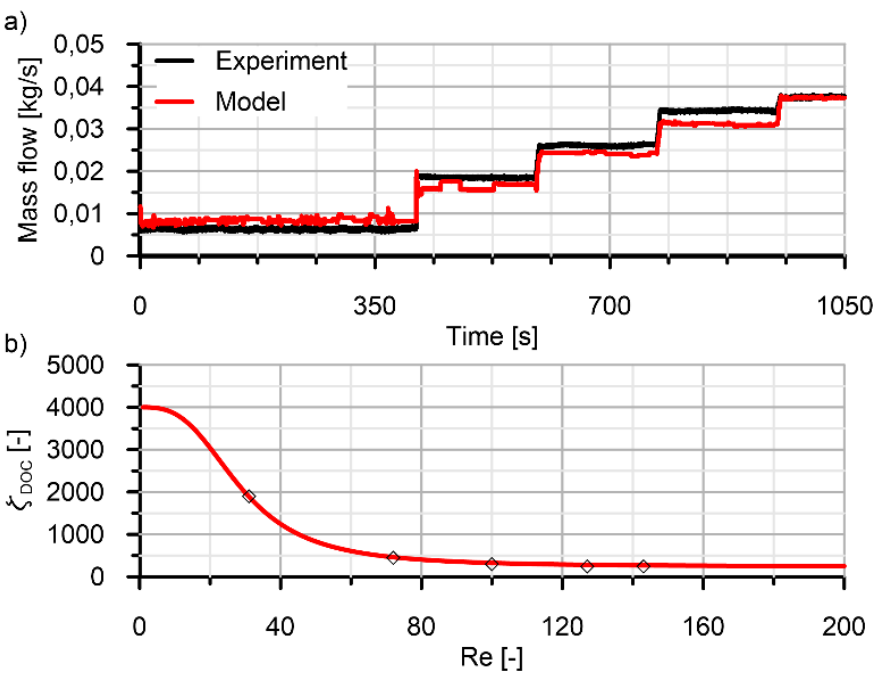

Figure 9. Pressure drop coefficient determination and mass flow prediction in motoring tests.

Page 11 of 22
In the case of $\mathrm{HC}$, the conversion efficiency is given by the combination of oxidation and accumulation on an absorbent material, typically a zeolite [60]. Decane was used in this work as representative hydrocarbon of the Diesel engine emission [61,62], both for oxidation and storage modelling. Although the $\mathrm{HC}$ heat of adsorption is from one to two orders of magnitude lower than the heat of oxidation [63], it has been included in Eq. (50). The heat of adsorption is dependent on the temperature and the zeolite formulation [63]. Neglecting this last effect, the HC storage was modelled as a physisorption so that the heat of adsorption was approximated to the heat of condensation $[64,65]$.

The integration along the monolith length of the $\mathrm{CO}$ and $\mathrm{HC}$ conservation equations in the gas bulk phase (Eq. (37)) and within the washcoat (Eq. (38)) gives as a result the outlet molar fraction as shown in Eqs. (71) and (72). In order to obtain an explicit solution for pollutants outlet concentration, which contributes to reduce the computational effort, the $\mathrm{O}_{2}$ concentration is assumed constant along the monolith. In addition, the inhibition terms are assumed to be dependent on the chemical species concentration in the washcoat obtained at the end of the previous time-step.

$$
\begin{gathered}
X_{C O, \text { out }}=X_{C O, \text { in }} e^{\left(1-\frac{4}{\pi}\right) \frac{k_{\text {ox }, C O} \eta_{\text {int }, o x, C o b_{1} X_{O_{2}}}}{G_{o x, C O}}} \\
X_{H C, \text { out }}=\frac{\left(b_{3}+b_{2} X_{H C, \text { in }}\right) e^{b_{2} \tau}-b_{3}}{b_{2}}
\end{gathered}
$$

The parameter $b_{1}$ in Eq. (71) is given by

$$
b_{1}=\frac{k_{m, C O}}{\frac{\alpha}{\pi}\left(1-\frac{\pi}{4}\right) \frac{k_{o x, C O} \eta_{i n t, o x, C O} X_{O_{2}}}{G_{o x, C O}}+k_{m, C O}}
$$

and parameters $b_{2}$ and $b_{3}$ present in HC solution (Eq. (72)) are defined as

$$
\begin{gathered}
b_{2}=\left(1-\frac{4}{\pi}\right)\left(k_{a d s, H C} \eta_{i n t, a d s, H C}(1-\theta) \psi+\frac{k_{o x, H C} \eta_{i n t, o x, H C} X_{O_{2}}}{G_{o x, H C}}\right) \\
\frac{k_{m, H C} X_{H C}}{\frac{\alpha}{\pi}\left(1-\frac{\pi}{4}\right)\left(\eta_{i n t, a d s, H C} k_{a d s, H C}(1-\theta) \psi+\frac{k_{o x, H C} \eta_{i n t, o x, H C} X_{O_{2}}}{G_{o x, H C}}\right)+k_{m, H C}}(74) \\
b_{3}=\left(\frac{4}{\pi}-1\right)\left(\eta_{i n t, d e s, H C} k_{d e s, H C} \theta \psi\right. \\
\left.-\left(\eta_{\text {int }, a d s, H C} k_{a d s, H C}(1-\theta) \psi+\eta_{i n t, o x, H C} \frac{k_{o x, H C}}{G_{o x, H C}} X_{O_{2}}\right) b_{4}\right)
\end{gathered}
$$

being $b_{4}$ :

$$
b_{4}=\frac{\eta_{\text {int }, d e s, H C} k_{d e s, H C} \theta \psi}{\eta_{i n t, a d s, H C} k_{a d s, H C}(1-\theta) \psi+\eta_{i n t, o x, H C} \frac{k_{o x, H C} X_{O_{2}}}{G_{o x, H C}}+\frac{\pi k_{m, H C}}{\alpha\left(1-\frac{\pi}{4}\right)}}
$$

The steady-state tests were run at $1500 \mathrm{rpm}, 2000 \mathrm{rpm}$ and $2500 \mathrm{rpm}$ varying the engine load from $5 \%$ to $40 \%$ in order to cover the low to medium exhaust temperature range. The engine operation was continuously monitored to account for thermal transient effects. Figure 10 compares experimental and modelling results concerning outlet gas and canning surface temperature in steady-state tests at $2500 \mathrm{rpm}$. Every step in temperature identifies a change in engine load. The inlet DOC gas temperature is represented by the dashed black series in plot (a). Being the surface temperature measured at the 
middle of the DOC monolith, the model shows good ability to predict the thermal response of the device, both in monolith and canning.

The outlet gas temperature prediction is sensitive to its increase over inlet gas temperature at low engine load because of the heat released by the $\mathrm{CO}$ and $\mathrm{HC}$ oxidation.
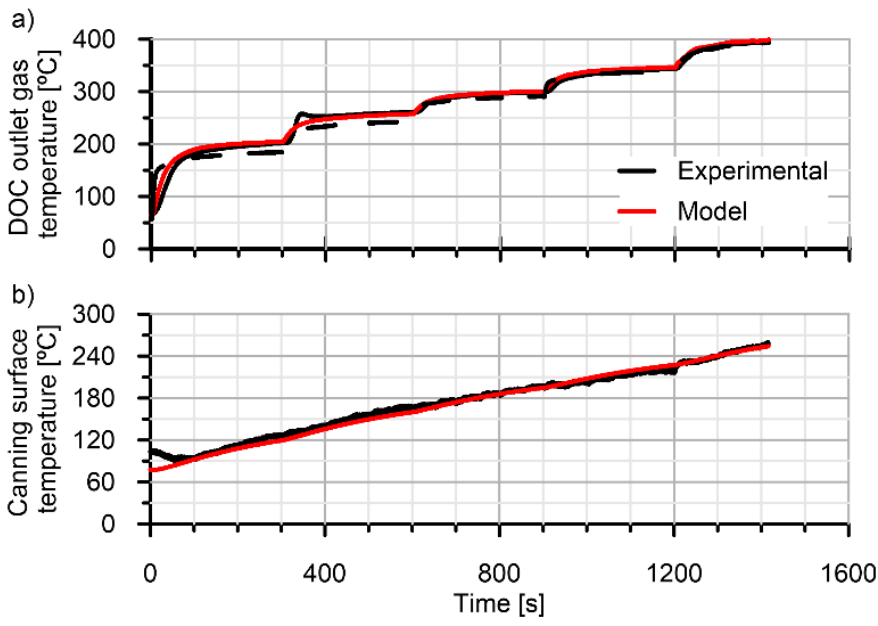

Figure 10. Comparison between experimental and modelled DOC outlet gas and canning surface temperature at $2500 \mathrm{rpm}$ with engine load steps from 5\% to $40 \%$.

Regarding this aspect, Figure 11 represents the conversion efficiency of $\mathrm{CO}$ and $\mathrm{HC}$ corresponding to steady-state points at different engine speeds. The calibration of $\mathrm{CO}$ and $\mathrm{HC}$ kinetic constants parameters is shown in Table 3. Figure 11(a) shows the DOC conversion efficiency when the engine was moved from motoring to $5 \%$ in engine load at $1500 \mathrm{rpm}$. It is shown how the $\mathrm{CO}$ conversion efficiency grows during the first seconds due to the progressively increase of the inlet temperature. However, $\mathrm{HC}$ conversion efficiency is high from the very beginning due to $\mathrm{HC}$ adsorption at low temperature. The model captures properly both the oxidation and adsorption processes providing a good prediction of the conversion efficiency for both pollutants. Plots (b) and (c) in Figure 11 confirm the good ability of the model to determine the conversion efficiency as the temperature increases what makes the conversion efficiency be progressively limited by mass transfer and pore diffusion processes. Although both $\mathrm{CO}$ and $\mathrm{HC}$ conversion efficiencies are slightly overestimated, the results are very close to the experimental data being even noticed the $\mathrm{HC}$ conversion sensitivity to change in the operating point in Figure 11(c).

As a final step, the calibrated DOC model was applied to the simulation of a WLTC run at ambient temperature. DOC and DPF were modelled together being the DPF model calibration described in next section. From a thermo-and fluid-dynamic point of view, Figure 12 shows a good prediction of the mass flow and DOC outlet gas temperature. Some deviations are observed in mass flow, mainly during sudden accelerations. In the case of the outlet gas temperature, the predicted profile at the DOC outlet is very accurate but a faster response of the model is noticed, especially during the warm-up period. On the one hand, part of the discrepancies seem to be due to a pure delay in the experimental signal, which is probably related to the characteristic thermocouple response. On the other hand, the inherent limitations of a lumped model seem also to be present. During the warm-up period, the outlet gas temperature is overpredicted by the model. It is not related to the higher $\mathrm{HC}$ conversion efficiency predicted by the model (see Figure 13), since during this phase hydrocarbons are adsorpted but not oxidized.

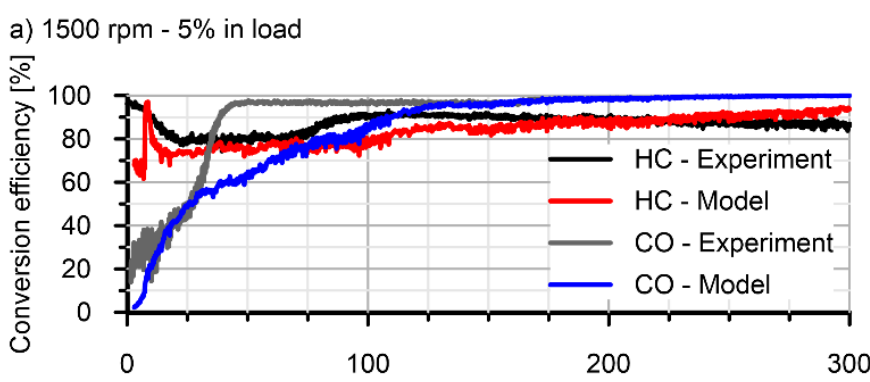

b) $2000 \mathrm{rpm}-10 \%$ in load

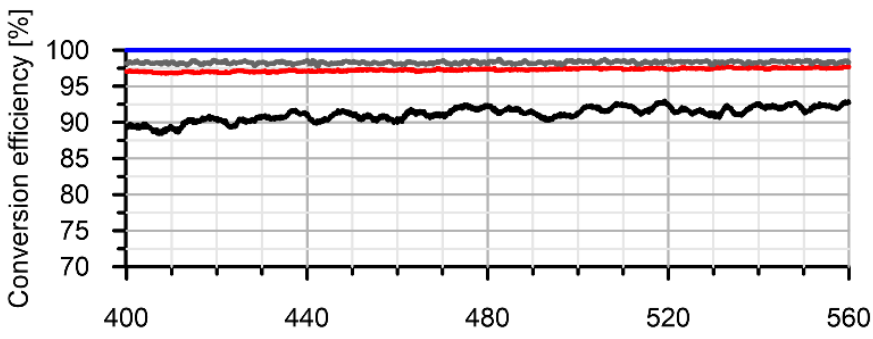

c) $2500 \mathrm{rpm}-20 \% \& 40 \%$ in load

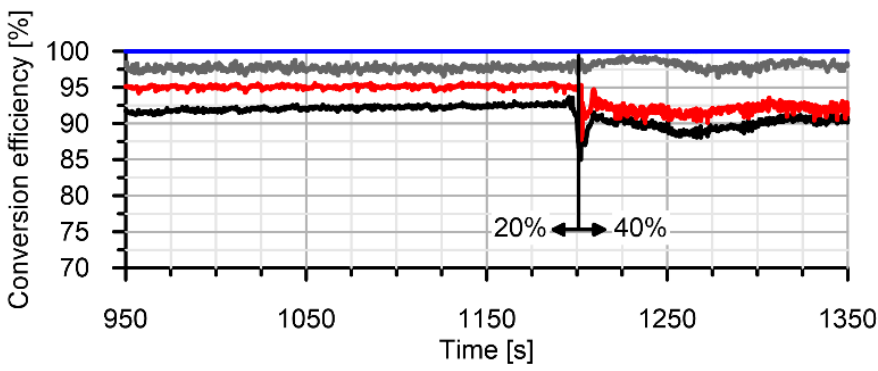

Figure 11. Comparison between experimental and modelled HC and CO conversion efficiency during thermal stabilization of different steady-state operating conditions.

Table 3. DOC kinetic constants definition.

\begin{tabular}{|c|c|c|}
\hline Reactions & $\mathbf{P}_{\mathrm{f}}\left[\mathrm{s}^{-1}\right]$ & $\mathbf{E}_{\mathrm{a}}[\mathrm{J} / \mathrm{mol}]$ \\
\hline HC adsorption & 0.2 & 0 \\
\hline HC desorption & 50000 & 107500 \\
\hline HC oxidation & $5 \times 10^{15}$ & 68000 \\
\hline CO oxidation & $2.5 \times 10^{17}$ & 80000 \\
\hline \multicolumn{3}{|c|}{ Inhibition terms } \\
\hline \multicolumn{3}{|c|}{$G_{o x, H C}=T_{i n}\left(1+K_{1} X_{C O, S}+K_{2} X_{H C, S}\right)^{2}\left(1+K_{3} X_{C O, S}^{2} X_{H C, S}^{2}\right)\left(1+K_{4} X_{N O, S}^{0.7}\right)$} \\
\hline \multicolumn{3}{|c|}{$G_{o x, C O}=T_{i n}\left(1+K_{5} X_{C O, S}+K_{6} X_{H C, S}\right)^{2}\left(1+K_{7} X_{C O, S}^{2} X_{H C, S}^{2}\right)\left(1+K_{8} X_{N O, S}^{0.7}\right)$} \\
\hline & $\mathbf{P}_{\mathrm{f}}[-]$ & $\mathrm{E}_{\mathrm{a}}[\mathrm{J} / \mathrm{mol}]$ \\
\hline$K_{1}$ & 555 & -7990 \\
\hline$K_{2}$ & $1.58 \times 10^{3}$ & $-3 \times 10^{3}$ \\
\hline$K_{3}$ & 2.98 & -96534 \\
\hline$K_{4}$ & $4.79 \times 10^{5}$ & 31036 \\
\hline$K_{5}$ & 555 & -7990 \\
\hline$K_{6}$ & $1.58 \times 10^{3}$ & $-3 \times 10^{3}$ \\
\hline$K_{7}$ & 2.98 & -96534 \\
\hline$K_{8}$ & $4.79 \times 10^{5}$ & 31036 \\
\hline
\end{tabular}

Page 12 of 22 
Therefore, the most probable cause of the outlet gas temperature overprediction is the decrease of the heat transfer rate with respect to the actual behavior. The lumped modelling involves that the monolith is heated homogenously. As a result, the local effects cannot be captured. In real operation, these are very relevant since only the entering region of the monolith is heated up significantly during the beginning of the warm-up phase. It leads to a dynamic process governed by heat transfer in axial and radial directions, which is neglected by the lumped approach.

The results in Figure 12(b) allow also noting that the maximum rate of outlet gas temperature variation is about $150^{\circ} \mathrm{C}$ in $100 \mathrm{~s}$ (order of magnitude) during the WLTC. Applying a time-step of $0.02 \mathrm{~s}$ (typical of ECU), it means that the maximum temperature variation per timestep is about $0.03{ }^{\circ} \mathrm{C}$. The order of magnitude of the time-step in the case of $1 \mathrm{D}$ gas dynamics software is $10^{-5} \mathrm{~s}$. It means that the maximum temperature variation per time-step is around $1.5 \times 10^{-5}{ }^{\circ} \mathrm{C}$. These results demonstrate that the isothermal condition hypothesis to calculate the internal pore diffusion efficiency, which is discussed next, can be accepted in this application case.

a)
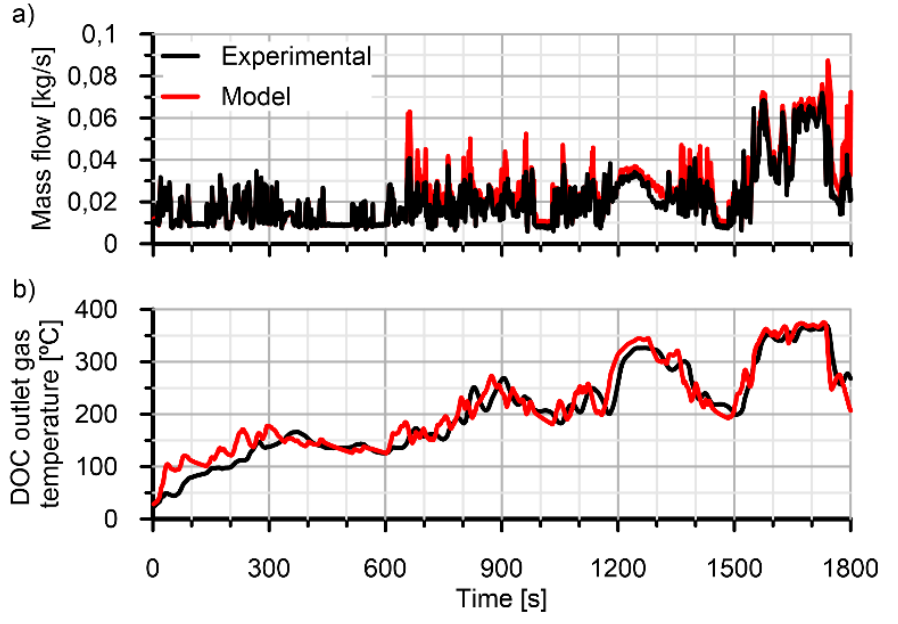

Figure 12. Comparison between experimental and modelled DOC mass flow and outlet gas temperature during driving conditions (WLTC).

Concerning pollutant emissions, Figure 13 represents in plots (a) and (b) the comparison between experimental and modelled accumulated conversion efficiency for $\mathrm{HC}$ and $\mathrm{CO}$ respectively. For the sake of completeness, Figure 14 shows the experimental and modelled cumulative emissions of $\mathrm{HC}$ and $\mathrm{CO}$ at the DOC inlet and outlet. The series are normalized with respect to the experimental accumulated emission of every species at the DOC inlet at the end of the WLTC.

Again, high accuracy is obtained during the whole WLTC, which requires good sensitivity to temperature, in terms of oxidation but also adsorption and desorption of $\mathrm{HC}$, pore diffusion and mass transfer effects. Nevertheless, it is important to note again that the kinetic constants are computed with the inlet gas temperature instead of the substrate wall temperature, which is the governing one. As previously described, it is done both in the gaseous emissions modelling (DOC) and in the DPF regeneration sub-model. This assumption is useful in terms of computational effort. In the case of OBD applications, the inlet gas temperature can be easily measured and allows decoupling chemical computations from heat transfer modelling. In addition, the sub-models can also be decoupled even considering radial heat transfer modelling in the monolith. If the substrate wall temperature was used in the kinetic constant calculation, every radial region of the monolith would require to be calculated separately for every sub-model. Consequently, the computational effort would be increased.
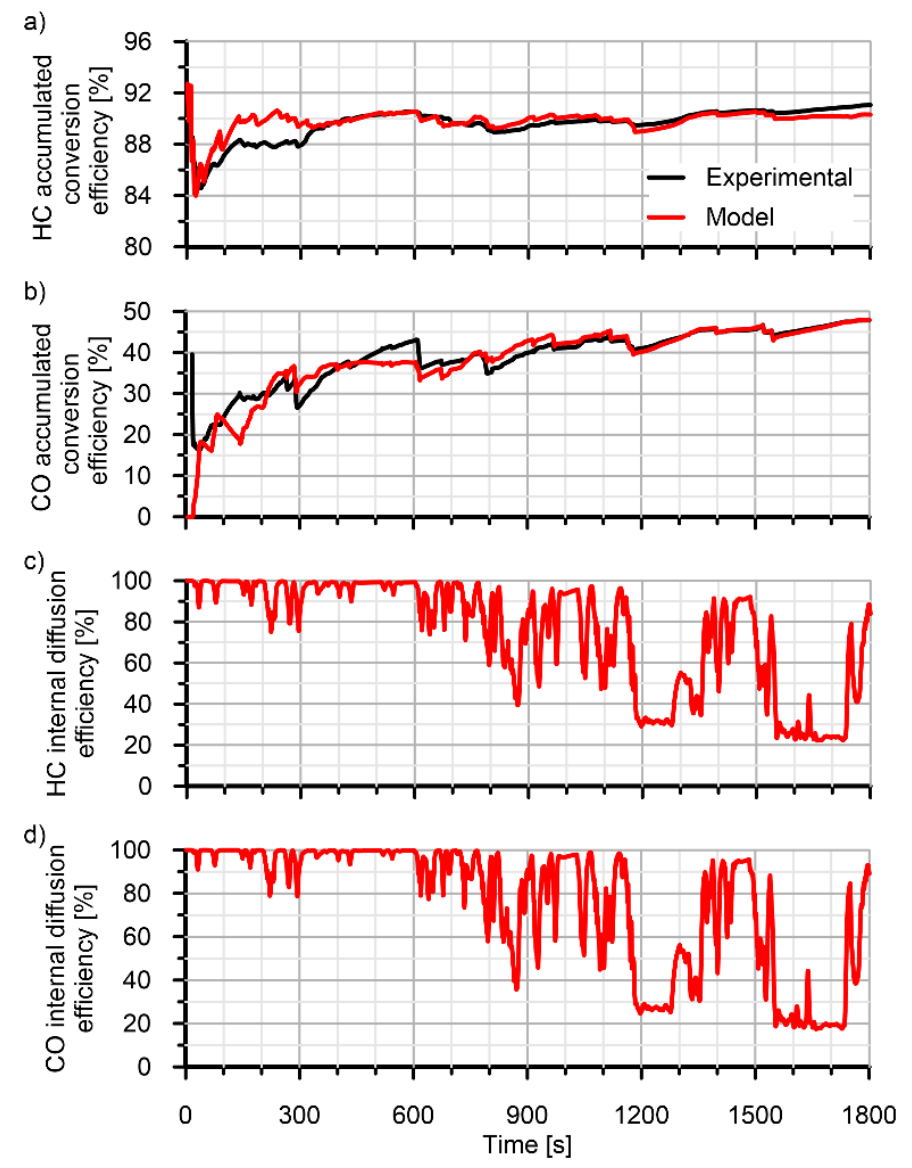

Figure 13. HC and $\mathrm{CO}$ accumulated conversion efficiency and internal diffusion efficiency during WLTC.

a)

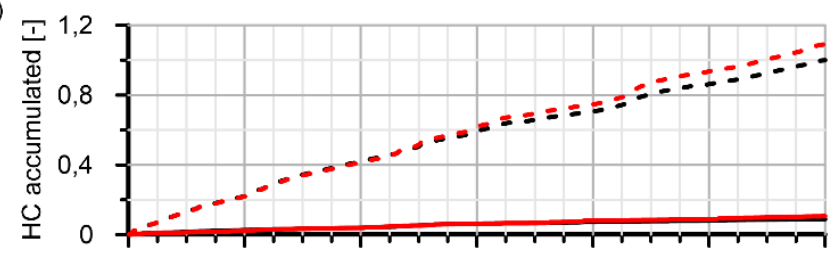

b)

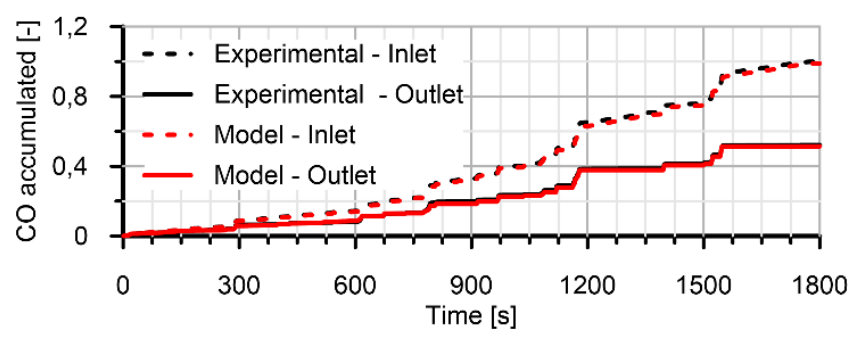

Figure 14. HC and CO cumulative emissions during WLTC.

Nevertheless, the use of the inlet gas temperature can lead to lower model accuracy when the difference between the gas and the substrate temperatures is high. It is demonstrated in Figure 13(a). The $\mathrm{HC}$ accumulated conversion efficiency is overestimated during the first cycle phase. It is because the inlet gas temperature is clearly higher than the substrate temperature, which is progressively 
increasing from ambient conditions governed by the substrate thermal inertia.

Pore diffusion and mass transfer take place simultaneously during the high and extra-high speed cycle phases. Thus, it is possible to observe a drop of accumulated conversion efficiency both in $\mathrm{HC}$ and $\mathrm{CO}$ at $1170 \mathrm{~s}$ and $1550 \mathrm{~s}$ in the experiment and the model. These sudden drops are related to instantaneous slip emissions caused by a fast mass flow increase (Figure 12(a)), which leads to the residence time reduction, and a decrease of the internal diffusion efficiency up to $20 \%$, as plotted in Figure 13(c) and (d). It is due to the temperature increase, which is not enough to compensate these negative effects by increasing the kinetic constant.

$\mathrm{HC}$ adsorption and desorption are evidenced separately in Figure 15 and Figure 16. Figure 15 depicts the low speed WLTC phase during which adsorption is the dominant phenomena abating $\mathrm{HC}$ emissions. Figure 15(a) shows the inlet $\mathrm{HC}$ molar fraction and its variation due to oxidation and adsorption processes (negative values). As observed, oxidation and adsorption are complementary but the adsorption takes more importance during this phase because of the low temperature periods ((Figure 15(b)). Consequently, it contributes to high accumulated $\mathrm{HC}$ conversion efficiency during the very WLTC beginning and leads the surface coverage to increase (Figure 15(d)).

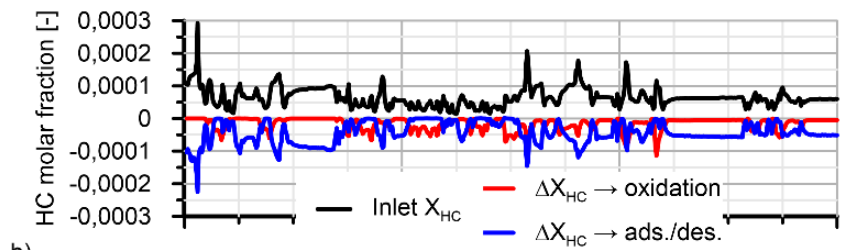

b)

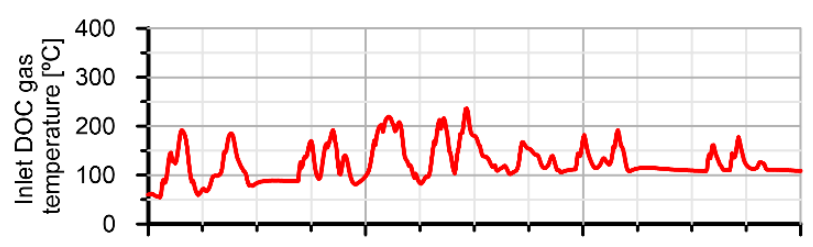

c)

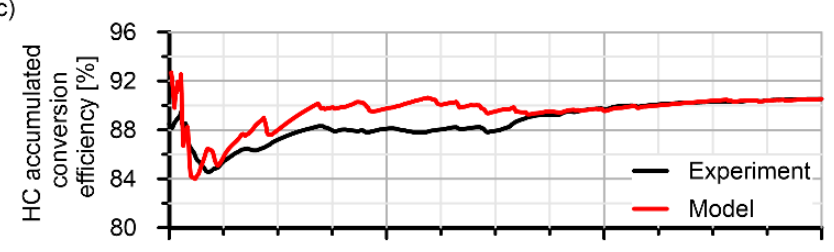

d)

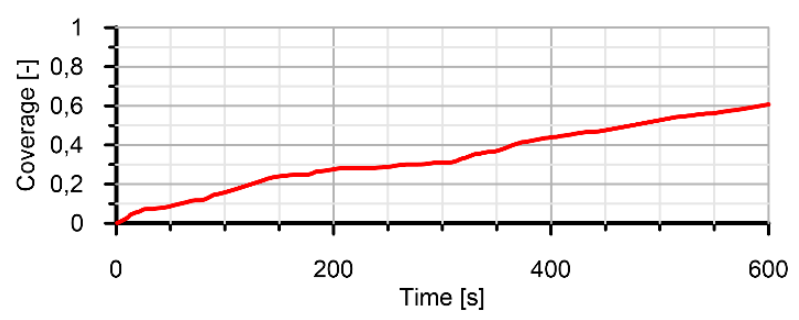

Figure 15. Hydrocarbon adsorption phenomena during low speed phase of WLTC.

After the low speed phase, the surface coverage keeps almost constant because of the high oxidation rate until the desorption starts. Figure 16 shows the $\mathrm{HC}$ related variables during the high-speed WLTC phase. First HC desorption event occurs at $1120 \mathrm{~s}$ (Figure 16(a)). Although the model shows a decrease in HC accumulated conversion efficiency more marked than in experimental results, the model captures the following positive slope in this variable like in the experiment. This positive slope points out the oxidation of a high $\mathrm{HC}$ mass that can be only explained by the combustion of the incoming and the progressively desorpted $\mathrm{HC}$ before it leaves the DOC monolith. This process produces the gradual decrease of the surface coverage while the temperature is kept over $300^{\circ} \mathrm{C}$. According to these results, the tested DOC is able to adsorp $\mathrm{HC}$ below $150^{\circ} \mathrm{C}$, keeping them retained until $300^{\circ} \mathrm{C}$ are reached. This temperature for desorption ensures high oxidation rate and avoids excessive HC slip.

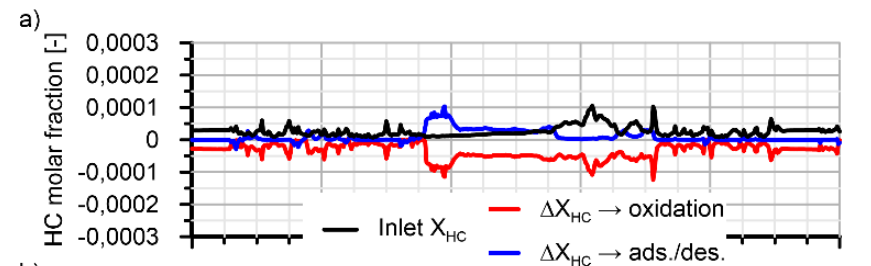

b)

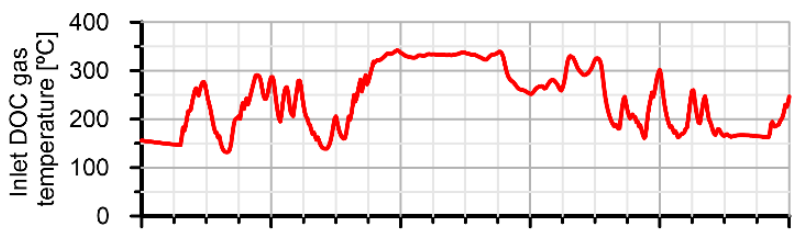

c)

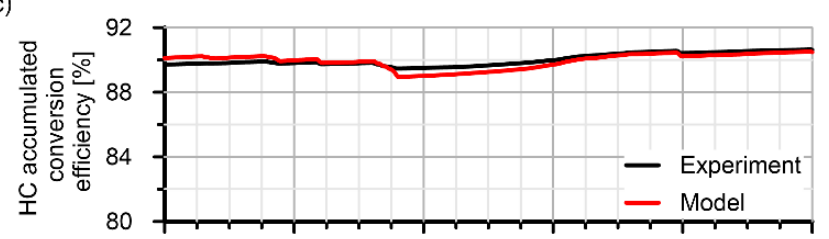

d)

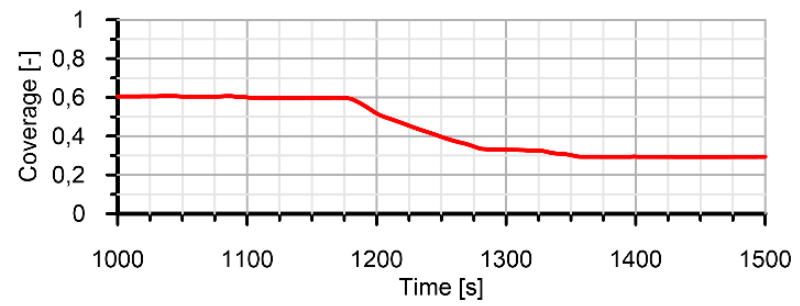

Figure 16. Hydrocarbon desorption phenomena during high-speed phase of WLTC.

\section{DPF modelling}

The pressure drop characterization in wall-flow particulate filters requires several steps to distinguish between clean and loading conditions. In clean conditions, the DPF pressure drop to volumetric flow rate ratio is a linear function of the volumetric flow rate [30], as shown in Figure 17(a). These results correspond to pressure drop measurements in a flow test rig according to the procedure described in [66]. Expanding Eq. (2), the term $c_{1}$ in Eq. (77), which is obtained experimentally, results a function of the monolith geometry, the dynamic viscosity and the porous wall permeability, as observed in Eq. (78). Therefore, the porous wall permeability can be directly calculated from the experimental. A value of $7.64 \times 10^{-13} \mathrm{~m}^{2}$ was obtained for the tested DPF. Next, the inertial pressure drop coefficient $\left(s_{t}\right)$, which is the only remaining unknown parameter in Eq. (79), can be calibrated from the pressure drop results. A constant pressure drop coefficient was imposed in this work. Figure 17(b) represents in red color the clean DPF pressure drop at ambient temperature conditions $\left(20^{\circ} \mathrm{C}\right)$ predicted by the model as a function of the mass flow. Despite the constant inertial pressure drop coefficient, results are well aligned with experimental data. It is due to the small

Page 14 of 22 
inertial pressure drop contribution at low mass flow in comparison to in-channel contributions [27].

$$
\begin{gathered}
\frac{\Delta p_{D P F}}{Q}=c_{1}+c_{2} Q \\
c_{1}=\frac{\mu}{2 V_{e f} \sigma}\left(\frac{w_{w}}{k_{w_{0}} \alpha}+\frac{8 F_{w} L_{e f}^{2}}{3 \alpha^{4}}\right) \\
c_{2}=\frac{2 \rho}{V_{e f}^{2} \sigma^{2} \alpha^{2}} s_{t}\left(\frac{L_{e f}}{\alpha}\right)^{2}
\end{gathered}
$$

a)

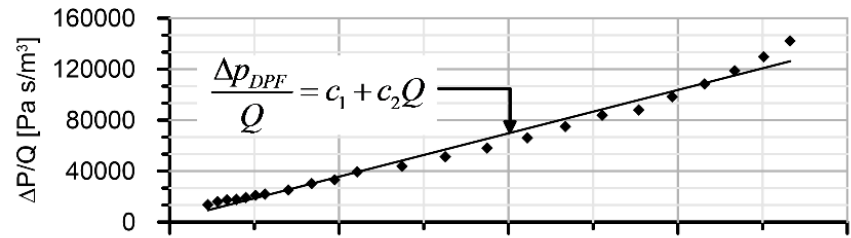

b)

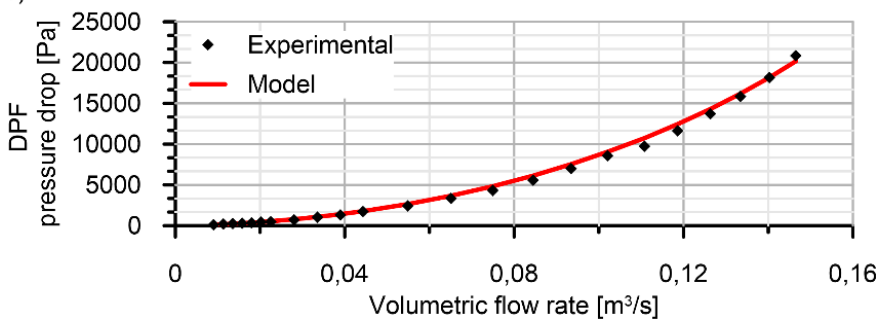

Figure 17. Clean DPF pressure drop characterization in flow test rig.

Once the clean porous wall permeability was determined, the porosity $(0.4)$ and the mean pore diameter $(21.4 \mu \mathrm{m})$ of the substrate were estimated considering Eq. (12) as described in [66].These data are needed to account for the change in pressure drop as filtration efficiency as the DPF is loaded. Thus, a soot loading test was performed under steady-state conditions. The operating point was selected to provide high opacity and low exhaust gas temperature at the DPF inlet to avoid passive regeneration. The DPF was weighed in hot conditions before and after the test to determine the final soot load [67]. This kind of test allows characterizing the pressure drop response and the filtration efficiency as a function of the soot load. As represented by red dots in Figure 18(a), a set of discrete conditions test were selected along the soot loading. The points in deep bed filtration regime were used to calibrate the shape factor, whose fitted correlation is shown in Eq. (80), and the soot thickness penetration fraction into the porous wall $(2.95 \%)$. The last governs the pressure drop during this regime and defines the saturation soot mass.

$$
\chi=3.011\left(\Phi_{\rho_{s}}\right)^{-0.986}+0.0149
$$

The pressure drop during the cake filtration regime is conditioned by the particulate layer porosity, which was found to be 0.66 when imposing the mode of the particle size distribution $(80 \mathrm{~nm})$ as collector diameter to set the particulate layer permeability according to Eq. (25).

Figure 19(a) shows the mass flow prediction as a function of the soot load when the experimental pressure drop was imposed applying the shape factor given by Eq. (80). The mass flow, which was almost constant, is properly predicted throughout the test as well as the soot

Page 15 of 22 load (Figure 19(b)). In fact, the soot load is a function of the mass flow but also depends on the computation of the filtration efficiency, whose evolution until the maximum value is zoomed in Figure 19(c). Finally, Figure 19(d) demonstrates that the model also accounts properly for heat transfer. An accurate prediction of the DPF outlet gas temperature is provided both during the thermal transient period and once steady-state conditions are reached.

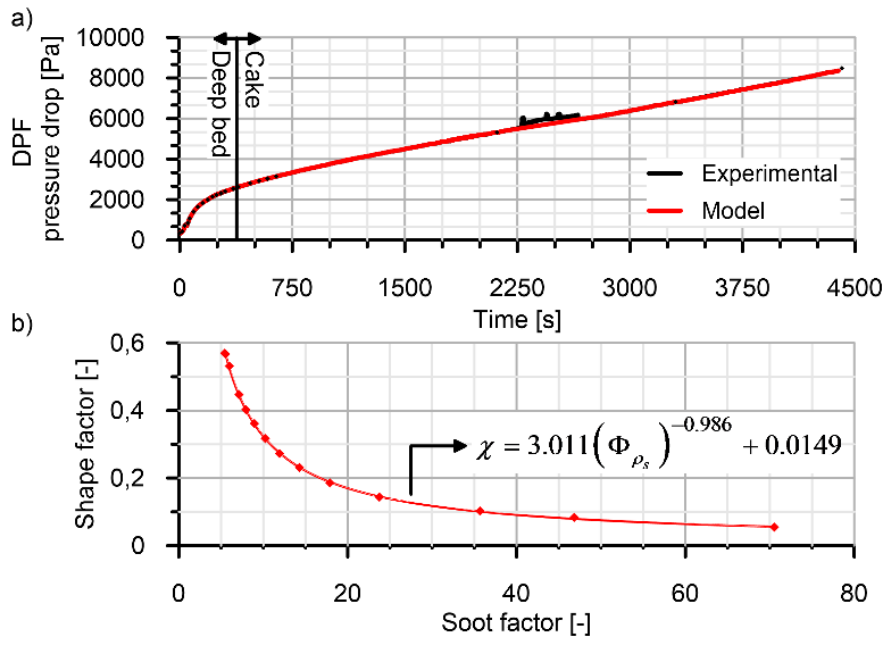

Figure 18. Shape factor correlation for DPF pressure drop prediction.

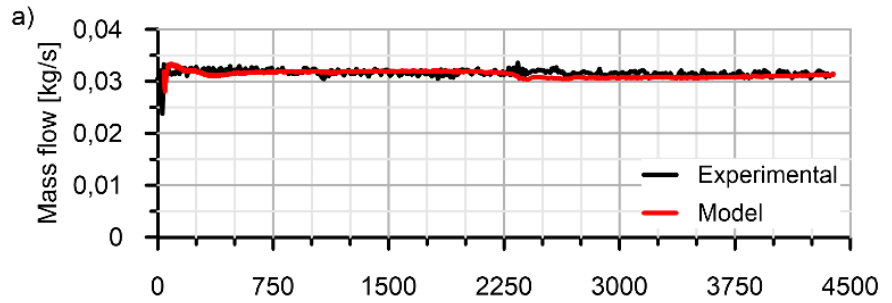

b)
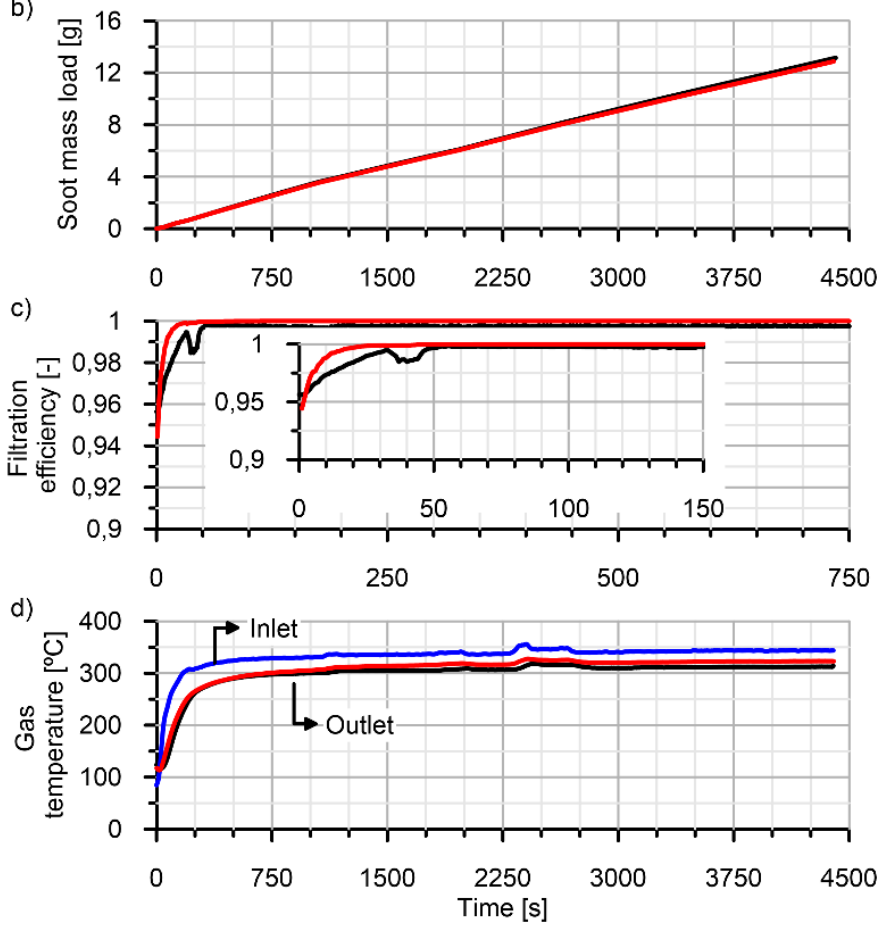

Figure 19. Comparison between DPF experimental and model response during the soot loading test under steady-state conditions. 
The soot oxidation was firstly calibrated by modelling an active regeneration process, which is summarized in Figure 20. It was performed after the soot loading test so that the initial substrate conditions are known from the end modelling shown in Figure 19. Again, the DPF pressure drop is imposed as boundary condition so that the fluid-dynamic model calculates the mass flow across the DPF. Being the mass flow constant, the time-dependence of the pressure drop is governed by the soot load and gas temperature, which are both a function of the soot oxidation rate. It is defined by the soot oxidation kinetic constant as well as the equilibrium adsorption/desorption constant for every oxidizing species, whose parameters in the Arrhenius type equation are detailed in Table 4. The difference between the pre-exponential factor for soot oxidation in the cake and the porous wall is mainly due to the different soot deposition pattern in these two regions. The model is solved in the tangential direction of the porous medium. Therefore, the amount of soot in the particulate layer per unit of length is higher in this region than in the porous wall, where there are soot and ceramic substrate. According to Eq. (53), the model accounts for soot properties by means of the soot geometric surface area. The value proposed by Bisset in [9] is used without distinction between the porous medium regions. However, this value must be corrected in the porous wall because of the soot and unit collectors interaction. Formally, this correction is falling into the pre-exponential factor calibration.

a)

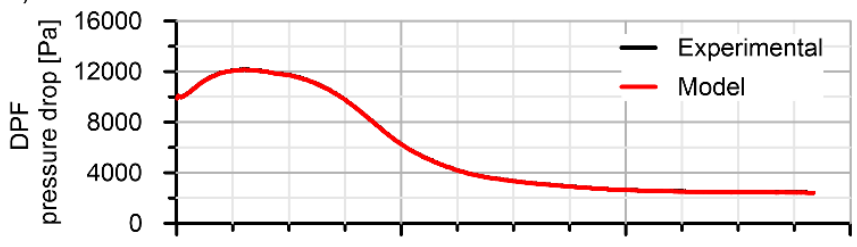

b)

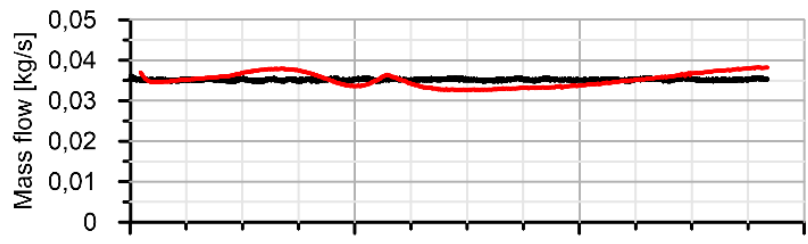

c)

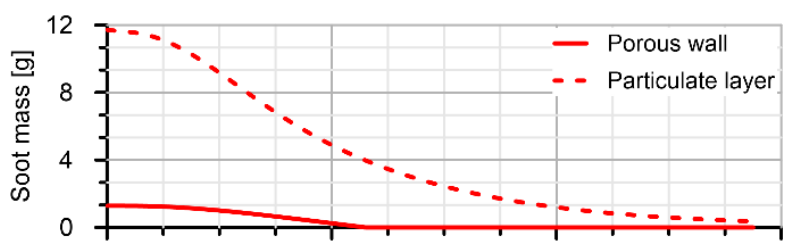

d)

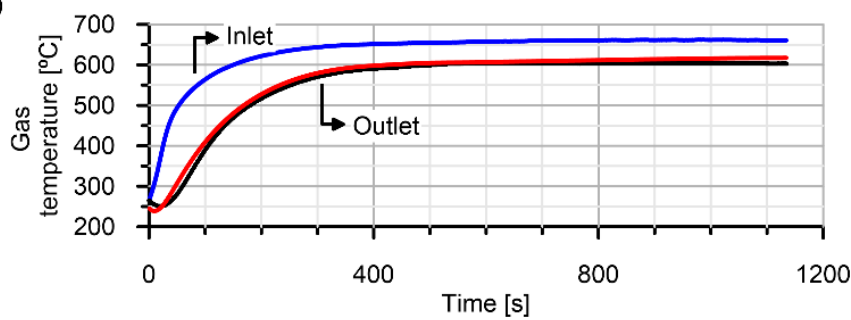

Figure 20. Comparison between DPF experimental and model response during active regeneration test.

This way, Figure 20(c) represents the soot mass load prediction into the DPF distinguishing between porous wall and particulate layer throughout the regeneration process. The corresponding soot depletion rate governs the heat release rate determining the outlet gas temperature, which is plotted in Figure 20(d).

Table 4. Kinetic constant parameters for the DPF regeneration sub-model.

\begin{tabular}{|l|l|c|c|c|c|}
\hline \multicolumn{2}{|c|}{} & $\begin{array}{c}\text { Oxidation } \\
\mathrm{P}_{\mathrm{f}}[\mathrm{m} / \mathrm{s}]\end{array}$ & $\begin{array}{c}\text { Oxidation } \\
\mathrm{E}_{\mathrm{a}} \\
{[\mathrm{J} / \mathrm{mol}]}\end{array}$ & $\begin{array}{c}\text { Equilibrium } \\
\text { ads/des } \mathrm{P}_{\mathrm{f}} \\
{[-]}\end{array}$ & $\begin{array}{c}\text { Equilibrium } \\
\text { ads/des } \Delta \mathrm{H} \\
{[\mathrm{J} / \mathrm{mol}]}\end{array}$ \\
\hline $\begin{array}{l}\text { Particulate } \\
\text { layer }\end{array}$ & $\mathrm{O}_{2}$ & 7.2 & 108500 & 0.000033 & -85000 \\
\cline { 2 - 6 } & $\mathrm{NO}_{2}$ & 30000 & 100000 & 0.0001 & -75000 \\
\hline $\begin{array}{l}\text { Porous } \\
\text { wall }\end{array}$ & $\mathrm{O}_{2}$ & 0.33 & 108500 & 0.000033 & -85000 \\
\cline { 2 - 7 } & $\mathrm{NO}_{2}$ & 1500 & 100000 & 0.0001 & -75000 \\
\hline
\end{tabular}

This model setup was finally applied to the modelling of the DPF response during WLTC driving conditions. The DOC model, whose performance has been discussed in the previous section, determined the DPF inlet flow conditions. As shown in Figure 21(a), the tailpipe gas temperature is predicted showing very good agreement with experimental data. In addition, Figure 21(b) shows the soot accumulation into the DPF, which began the driving cycle in clean conditions.

The soot accumulation governs the DPF pressure drop whose correct modelling is essential for the prediction of the mass flow across the aftertreatment devices, which has been already shown in Figure 12. In this test it depends on a proper definition of the clean substrate properties besides filtration efficiency and soot depletion rate as the cycle advances. It is interesting to note that a proper modelling of these phenomena is in turn dependent on the predicted mass flow in the previous time-step, since it sets the amount of soot mass entering the DPF. This fact demonstrates the need of a robust model calibration to avoid that the interaction among all sub-models might lead to progressive error increase. In addition, it means that a proper mass flow prediction is related to confident computation of other parameters such as the instantaneous accumulated soot mass. In Figure 21(b) the experimental filtrated soot mass, which was obtained from the inlet to outlet difference in soot mass flow measurements, shows very good agreement with the model results. The model slightly overestimates the filtrated soot mass according to the mass flow prediction shown in Figure 12 and the filtration efficiency results in Figure 19.

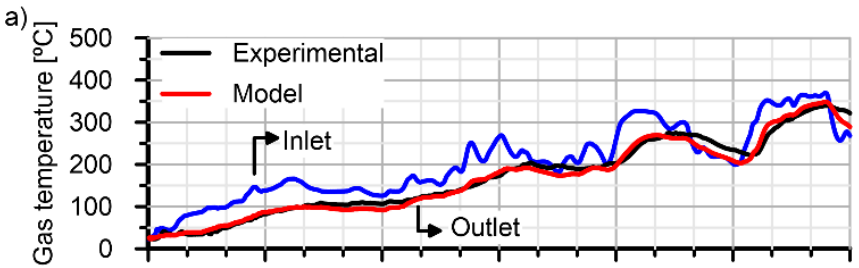

b)

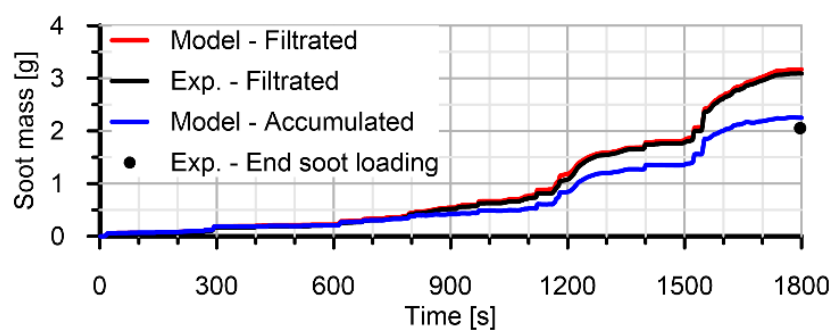

Figure 21. Comparison between experimental and modelled DPF outlet gas temperature and collected soot mass during WLTC. 
The experimental data are completed with the end soot load remaining into the DPF, which is based on DPF weighing data. The model provides the instantaneous amount of soot accumulated into the DPF, which is quantified as the difference between filtrated and regenerated soot mass. Since only the NOx concentration was measured, $80 \%$ of the $\mathrm{NO}_{2}$ to $\mathrm{NOx}$ ratio in equilibrium conditions was assumed in this test in order to define the $\mathrm{NO}_{2}$ concentration at the DPF inlet. It is shown that the predicted final amount of soot mass into the DPF is 2.25 $\mathrm{g}$, which is very close to the experimental value $(2.05 \mathrm{~g})$. This good agreement provides high confidence on the potential of the presented tool to explore thermal management and DPF design strategies to enhance the passive regeneration performance. These strategies might be based on the analysis of the flow properties and on the instantaneous amount of accumulated soot mass during the driving cycle, which is not possible to be obtained by experimental means.

\section{Real-time calculation assessment}

In order to demonstrate the capability of the proposed lumped models for real-time use, a computational effort assessment is presented in this section. The demand of DOC and DPF computational effort was evaluated in WLTC applying different time-steps ranging from representative ECU to gas dynamics software applications. Simulations were performed on a standard office PC with an Intel i7 processor and using Microsoft Visual Studio C++ release as programming and compiler environment. The results are presented in Table 5 normalized with respect to real-time. Similar results are obtained for both models, being slightly faster the DPF model. Three time-steps were selected to show the impact of the working routine environment on the computational effort. In gas dynamics software the time-step is governed by the CFL condition and the mesh size [68]. It leads to time-steps typically of 10-5 s in order of magnitude. Even imposing these characteristic time-steps, the DOC and DPF models are far below real-time requirements. As the time-step is increased towards typical ECU requirements, the computational effort gets around 3300 times faster than real-time. The quasi-steady approach of all sub-models and the lack of numerical stability criteria to be applied allows imposing the proposed time-steps in standalone executions (only the wall-flow and flow-through models, i.e. not integrated into gas dynamics software or similar). The only restriction is that the time-step must be lower than the input data updating time. These results strengthen the ability of the model to be used under different environments ranging from in-engine office simulation to hardware in-the-loop applications.

Table 5. Computational effort of DOC and DPF lumped model in WLTC simulation referred to real-time.

\begin{tabular}{|l|c|c|}
\cline { 2 - 3 } \multicolumn{1}{c|}{} & \multicolumn{2}{c|}{ Normalized calculation time [-] } \\
\hline Time-step [s] & DOC & DPF \\
\hline $2 \times 10^{-2}$ & 0.0003 & 0.0003 \\
\hline $1 \times 10^{-4}$ & 0.0617 & 0.0542 \\
\hline $3 \times 10^{-5}$ & 0.2039 & 0.1875 \\
\hline
\end{tabular}

\section{Summary and conclusions}

A lumped exhaust aftertreatment modelling approach for throughflow and wall-flow monolithic reactors has been presented and described in this work. The main purpose has been to provide a model basis keeping physical and chemical content but ensuring low computational effort in different fields of use, such as on-board diagnostics, calibration or pre-design stages. In this sense, the model Page 17 of 22 accounts for a modular structure covering pressure drop, heat transfer and chemical processes as well as filtration efficiency and porous media micro-structure modelling in wall-flow monoliths. Pressure drop in flow-through monoliths is computed from a pressure drop coefficient that relies on experimental characterization. In wall-flow monoliths this approach is completed accounting for porous medium and friction pressure drop contributions separately. In case of the porous medium, the prediction capability is provided as a function of the soot mass loading. In fact, its influence is taken into account applying a packed bed of spherical particles approach to estimate the permeability from the filtration efficiency and, hence, the microstructural properties of the different porous media. The heat transfer is computed from the base of a lumped thermal scheme that accounts for the main heat fluxes as well as the thermal inertia of the substrate and canning materials. Finally, the chemical conversion in flowthrough monoliths is solved integrating the chemical species conservation equations into the bulk-gas phase and the washcoat along the monolith length. It is done including the assessment of the reaction rate limiting phenomena such as bulk mass transfer, internal pore diffusion and chemical kinetics. A similar procedure is applied to wall-flow monoliths regarding soot oxidation, which is modelled solving the oxidizing reactants conservation equations across the porous media in a three-layer approach.

The proposed models have been assessed applying them to the prediction of outlet flow properties in a close-coupled brick composed of a DOC and DPF. Experimental data were obtained from selected in-engine tests representing a simple but robust step-by-step calibration procedure. Finally, the model performance was assessed in a WLTC. All sub-models showed good and balanced accuracy to capture all relevant phenomena providing deeper understanding on the experimental results. It establishes a reliable basis for analysis and optimization of monolith functionalities, both concerning pollutants abatement and those related to engine integration. It is further strengthened by the assessment of the computational effort demand, which has been shown to be lower than real-time in all applications ranging from 5 times faster for typical gas dynamics code time-step to 3300 times faster for control and on-board control diagnostics proposals when run in standard office environment.

\section{References}

1. Regulation (EC) No 715/2007 of the European Parliament and of the Council of 20 June 2007 on type approval of motor vehicles with respect to emissions from light passenger and commercial vehicles (Euro 5 and Euro 6) and on access to vehicle repair and maintenance information, Official Journal of the European Union, June 2007, http://eur-lex.europa.eu/legalcontent/EN/ALL/?uri=CELEX\%3A32007R0715.

2. Johnson, T., Joshi, A., "Review of Vehicle Engine Efficiency and Emissions", SAE Technical Paper 2017-01-0907, 2017, doi: 10.4271/2017-01-0907.

3. Karsten, R., Grisstede, I., Franoschek, S., Seyler, M., et al., "Diesel NOx aftertreatment systems for upcoming LDVemission legislations", SAE ATA Convergence Conference, Torino, Italy, Nov. 2012.

4. Methe, H., Bunar, F., Scholz, R., et al., "The impact of MinNOx on powertrain development", $6^{\text {th }}$ MinNOx Conference, Berlin, $22^{\text {nd }}$ June 2016.

5. Badshah, H., Kittelson, D., Northrop, W., "Particle Emissions from Light-Duty Vehicles during Cold-Cold Start", SAE International Journal of Engines 9(3):1775-1785, 2016, doi: $\underline{10.4271 / 2016-01-0997 .}$. 
6. Chan, T.W., Saffaripour, M., Liu, F., Hendren, J., et al., "Characterization of real-time particle emissions from a gasoline direct injection vehicle equipped with a catalyzed gasoline particulate filter during filter regeneration", Emission Control Science and Technology 2:75-88, 2016, doi: 10.1007/s40825016-0033-3.

7. Wurzenberger, J.C., Kutschi, S., Bardubitzki, S., Fairbrother, R., et al., "Model based assessment of real-driving emissions - an office and hardware-in-the-loop simulation approach", 2016 CLEERS Workshop, Ann Arbor, USA, April 2016.

8. Nagar, N., He, X., Iyengar, V., Acharya, N., et al., "Real Time Implementation of DOC-DPF Models on a Production-Intent ECU for Controls and Diagnostics of a PM Emission Control System", SAE International Journal of Commercial Vehicles 2(2):222-233, 2009, doi:10.4271/2009-01-2904.

9. Bissett, E.J., "Mathematical model of the thermal regeneration of a wall-flow monolith diesel particulate filter", Chemical Engineering Science 39(7-8):1233-44, 1984, doi: 10.1016/00092509(84)85084-8.

10. Konstandopoulos, A.G., Johnson, J.H., "Wall-flow Diesel Particulate Filters -Their Pressure Drop and Collection Efficiency”, SAE Technical Paper 890405, 1989, doi: 10.4271/890405.

11. Depcik, C., Langness, C., Mattson, J., "Development of a Simplified Diesel Particulate Filter Model Intended for an Engine Control Unit", SAE Technical Paper 2014-01-1559, 2014, doi: $10.4271 /$ 2014-01-1559.

12. Depcik, C., "Combining the Classical and Lumpued Diesel Particulate Filter Models", SAE Technical Paper 2015-01-1049, 2015, doi: $10.4271 / 2015-01-1049$.

13. Wurzenberger, J.C., Bardubitzki, S., Kutschi, S., et al., "Modeling of Catalyzed Particulate Filters - Concept Phase Simulation and Real-Time Plant Modeling on HiL", SAE Technical Paper 2016-01-0969, doi: 10.4271/ 2016-01-0969.

14. Wurzenberger, J.C., Auzinger, G., Heinzle, R., Wanker, R., "1D Modelling of Reactive Fluid Dynamics, Cold Start Behavior of Exhaust Systems", SAE Technical Paper 2006-01-1544, 2006, doi: $10.4271 / 2006-01-1544$.

15. Wenzel, S., Despujols, B., Wahiduzzaman, S., Papadimitriou, I., "Development of Real Time Catalyst Model for Engine and Powertrain Control Design", SAE Technical Paper 2009-011273, 2009, doi: $10.4271 / 2009-01-1273$.

16. Guardiola, C., Pla, B., Piqueras, P., Mora, J., et al., "Modelbased passive and active diagnostics strategies for diesel oxidation catalysts", Applied Thermal Engineering 110:962-971, 2017, doi: 10.1016/j.applthermaleng.2016.08.207.

17. Galindo, J., Serrano, J.R., Piqueras, P., García-Afonso, Ó., "Heat transfer modelling in honeycomb wall-flow diesel particulate filters", Energy 43:201-213, 2012, doi: 10.1016/j.energy.2012.04.044.

18. Serrano, J.R., Climent, H., Piqueras, P., Angiolini, E., "Filtration modelling in wall-flow particulate filters of low soot penetration thickness", Energy 112:883-898, 2016 doi: 10.1016/j.energy.2016.06.121.

19. Serrano, J.R., Arnau, F.J., Piqueras, P., García-Afonso, Ó., "Packed bed of spherical particles approach for pressure drop prediction in wall-flow DPFs (diesel particulate filters) under soot loading conditions", Energy 58:644-654, 2013, doi: $\underline{\text { 10.1016/j.energy.2013.05.051 }}$

20. Martín, J., Arnau, F.J., Piqueras, P., Auñón, Á., "Development of an Integrated Virtual Engine Model to Simulate New Standard Testing Cycles", SAE Technical Paper 2018-01-1413, 2018.

21. Galindo, J., Tiseira, A., Navarro, R., Tarí, D., et al., "Compressor Efficiency Extrapolation for 0D-1D Engine

Page 18 of 22
Simulations", SAE Technical Paper 2016-01-0554, 2016, doi: 10.4271/2016-01-0554.

22. Serrano, J.R., Arnau, F.J., García-Cuevas, L.M., Dombrovsky, A. et al., "Development and validation of a radial turbine efficiency and mass flow model at design and off-design conditions", Energy Conversion and Management 125:281-293, 2016, doi: 10.1016/j.enconman.2016.09.032.

23. Torregrosa, A.J., Olmeda, P., Martín, J., Romero, C., "A tool for predicting the thermal performance of a Diesel engine", Heat Transfer Engineering 32(10):891-904, 2011, doi: 10.1080/01457632.2011.548639.

24. Broatch, A., Olmeda, P., Martín, J., Salvador-Iborra, J., "Development and Validation of a Submodel for Thermal Exchanges in the Hydraulic Circuits of a Global Engine Model", SAE Technical Paper 2018-01-0160, 2018.

25. Payri, F., Olmeda, P., Martin, J., Carreño, R., “A New Tool to Perform Global Energy Balances in DI Diesel Engines", SAE Int. J. Engines 7(1):43-59, 2014, doi: 10.4271/2014-01- 0665.

26. Masoudi, M., "Hydrodynamics of Diesel Particulate Filters", SAE Technical Paper 2002-01-1016, 2002, doi: 10.4271/200201-1016.

27. Torregrosa, A.J., Serrano, J.R., Arnau, F.J., Piqueras, P., “A fluid dynamic model for unsteady compressible flow in wallflow diesel particulate filters", Energy 36:671-684, 2011, doi: 10.1016/j.energy.2010.09.047.

28. Konstandopoulos, A.G., Kostoglou, M., Skaperdas, E., Papaioannou, E., et al., "Fundamental Studies of Diesel Particulate Filters: Transient Loading, Regeneration and Aging", SAE Technical Paper 2000-01-1016, 2001, doi: 10.4271/200001-1016.

29. Konstandopoulos, A.G., Skaperdas, E., Masoudi, M., "Inertial Contributions to the Pressure Drop of Diesel Particulate Filters", SAE Technical Paper 20001-01-0909, 2001, doi: 10.4271/ 200101-0909.

30. Konstandopoulos, A.G., "Flow Resistance Descriptors for Diesel Particulate Filters: Definitions, Measurements and Testing", SAE Technical Paper 2003-01-0846, 2003, doi: 10.4271/ 2003-01-0846.

31. Kladopoulou, E.A., Yang, S.L., Johnson, J.H., Parker, G.G., et al., "A Study Describing the Performance of Diesel Particulate Filters During Loading and Regeneration - A Lumped Parameter Model for Control Applications", SAE Technical Paper 2003-01-0842, 2003, doi: 10.4271/ 2003-01-0842.

32. Yapaulo, R.A., Wirojsakunchai, E., Orita, T., Foster, D.E. et al., "Impact of filtration velocities and particulate matter characteristics on diesel particulate filter wall loading", International Journal of Engine Research 10(5):287-304, 2009, doi: 10.1243/14680874JER03509.

33. Kamp, C., Sappok, A., Wong, V., "Soot and Ash Deposition Characteristics at the Catalyst-Substrate Interface and IntraLayer Interactions in Aged Diesel Particulate Filters Illustrated Using Focused Ion Beam (FIB) Milling", SAE Technical Paper 2012-01-0836, doi: 10.4271/2012-01-0836.

34. Lapuerta, M., Ballesteros, R., Martos, F.J., "A method to determine the fractal dimension of diesel soot agglomerates", Journal of Colloid and Interface Science 303(1):149-158, 2006, doi: 10.1016/j.jcis.2006.07.066.

35. Neer A., Koylu, U.O., "Effect of operating conditions on the size, morphology, and concentration of submicrometer particulates emitted from a Diesel engine", Combustion and Flame 146(1-2):142-154, 2006, doi: 10.1016/j.combustflame.2006.04.003.

36. Konstandopoulos, A.G., Kostoglou, M., Vlachos, N., Kladopoulou, E., "Progress in Diesel Particulate Filter 
Simulation”, SAE Technical Paper 2005-01-0946, 2005, doi: 10.4271/2005-01-0946.

37. Lapuerta, M., Oliva, F., Martínez-Martínez, S., "Modeling of the Soot Accumulation in DPF Under Typical Vehicle Operating Conditions", SAE International Journal of Fuels and Lubricants 3(2):532-542, 2010, doi: 10.4271/2010-01-2097.

38. Lee, K.W., Gieseke, J.A., "Collection of aerosol particles by packed beds", Environmental Science Technology 13(4):466470, 1979, doi: 10.1021/es60152a013.

39. Serrano, J.R., Bermúdez, V., Piqueras, P., Angiolini, E., "On the impact of DPF downsizing and cellular geometry on filtration efficiency in pre- and post-turbine placement", Journal of Aerosol Science 113:20-35, 2017, doi: 10.1016/j.jaerosci.2017.07.014.

40. Logan, B.E., Jewett, D.G., Arnold, R.G., Bouwer, E.J., O'Melia, C.R., "Clarification of clean-bed filtration models", Journal of Environmental Engineering 121(12):869-873, 1995, doi: 10.1061/(ASCE)0733-9372(1995)121:12(869).

41. Choi, S., Lee, K., "Detailed investigation of soot deposition and oxidation characteristics in a diesel particulate filter using optical visualization", SAE Technical Paper 2013-01-0528, 2013, doi: 10.4271/2013-01-0528.

42. Kryl, D., Kočí, P., Kubíček M., Marek M., et al., "Catalytic converters for automobile Diesel engines with adsorption of hydrocarbons on zeolites", Industrial \& Engineering Chemistry Research 44:9524-9534, 2005, doi: 10.1021/ie050249v.

43. Hawthorn, R.D., "Afterburner catalysts effects of heat and mass transfer between gas and catalyst surface", AIChE Symposium Series 70:428-438, 1974.

44. Schejbal, M., Štěpánek, J., Kočí, P., Marek, M., Kubíček, M., "Sequence of monolithic converters DOC-CDPF-NSRC for lean exhaust gas detoxification: A simulation study", Chemical Engineering and Processing: Process Intensification 49(9):943952, 2010, doi: 10.1016/j.cep.2010.05.005.

45. Oh, S.H., Cavendish, J.C., "Transients of monolithic catalytic converters: response to step changes in feedstream temperature as related to controlling automobile emissions", Industrial and Engineering Chemistry Product Research and Development 21(1):29-37, 1982, doi: 10.1021/i300005a006.

46. Huo, W., Zhou, Z., Wang, F., Wang, Y., Yu, G., "Experimental study of pore diffusion effect on char gasification with $\mathrm{CO}_{2}$ and steam", Fuel 131:59-65, 2014, doi: 10.1016/j.fuel.2014.04.058.

47. Kočí, P., Štěpánek, F., Kubíček, M., Marek, M., "Modelling of micro/nano-scale concentration and temperature gradients in porous supported catalysts", Chemical Engineering Science 62(18):5380-5385, 2007, doi: 10.1016/j.ces.2006.12.033.

48. Van Donk, S., Janssen, A.H., Bitter, J.H., de Jong, K. P., "Generation, characterization, and impact of mesopores in zeolite catalysts", Catalysis Reviews, 45(2):297-319, 2003, doi:10.1081/CR-120023908.

49. Richards, P., "Fundamentals of chemical reaction engineering", Chapter 6, McGraw-Hill, New York, 2003, ISBN: 0-07-245007$\mathrm{X}$.

50. Fogler, H.S., "Elements of chemical reaction engineering", Prentice-Hall International, London, 1999, ISBN-10: 0133887510

51. Edelbauer, W., Kutschi, S., Wurzenberger, J.C., "xD+1D Catalyst Simulation - A Numerical Study on the Impact of Pore Diffusion”, SAE Technical Paper 2012-01-1296, 2012, doi: 10.4271/2013-01-0528.

52. Poling, B. E., Prausnitz, J. M., O'connell, J. P., "The properties of gases and liquids, Fifth edition", McGraw-Hill Education, New York, 2001, ISBN: 9780070116825.

53. Kandylas, I. P., Koltsakis, G.C., " $\mathrm{NO}_{2}$-assisted regeneration of diesel particulate filters: A modeling study", Industrial and

Page 19 of 22
Engineering Chemistry Research 41:2115-23, 2002, doi: 10.1021/ie010842m.

54. Maricq, M., Xu, N., "The effective density and fractal dimension of soot particles from premixed flames and motor vehicle exhaust", Journal of Aerosol Science 35(10):1251-1274, 2004, doi: 10.1016/j.jaerosci.2004.05.002.

55. Vander Wal, R.L., "Soot Nanostructure: Definition, Quantification and Implications", SAE Technical Paper 200501-0964, 2005, doi: 10.4271/2005-01-0964.

56. Messerer, A., Niessner, R., Pöschl, U., "Comprehensive kinetic characterization of the oxidation and gasification of model and real diesel soot by nitrogen oxides and oxygen under engine exhaust conditions: Measurement, Langmuir-Hinshelwood, and Arrhenius parameters", Carbon 44:307-24, 2006, doi: 10.1016/j.carbon.2005.07.017.

57. Dada, A. O., Olalekan, A.P., Olatunya, A. M., "Langmuir, Freundlich, Temkin and Dubinin-Radushkevich Isotherms Studies of Equilibrium Sorption of Zn2+ Unto Phosphoric Acid Modified Rice Husk", IOSR Journal of Applied Chemistry 3:2278-5736, 2012.

58. Torregrosa, A.J., Olmeda, P., Martín, J., Romero, C., "A tool for predicting the thermal performance of a Diesel engine", Heat Transfer Engineering 32(10):981-904, 2011, doi: 10.1080/01457632.2011.548639.

59. Payri, F., Olmeda, P., Arnau, F.J., Dombrovsky, A., Smith, L., "External heat losses in small turbochargers: Model and experiments", Energy 71:534-546, 2014, doi: 10.1016/i.energy.2014.04.096.

60. Kim, D.J., Kim, J.W., Yie, J.E., Moon, H., "Temperatureprogrammed adsorption and characteristics of honeycomb hydrocarbon adsorbers", Industrial and Engineering Chemistry Research 41(25):6589-6592, 2002, doi: 10.1021/ie020165b.

61. Siegl, W.O., Hammerle, R.H., Herrmann, H.M., Wenclawiak, B.W., et al., "Organic emissions profile for a light-duty diesel vehicle", Atmospheric Environment 33:797-805, 1999, doi: 10.1016/S1352-2310(98)00209-X.

62. Payri, F., Bermúdez, V.R., Tormos, B., Linares, W.G., "Hydrocarbon emissions speciation in diesel and biodiesel exhausts", Atmospheric Environment 43:1273-1279, 2009, doi: 10.1016/j.atmosenv.2008.11.029.

63. Dixit, L., Prasada Rao, T.S.R., "Heats of adsorption of hydrocarbons on zeolite surfaces: a mathematical approach", Journal of Chemical Information and Computer Sciences 39(2), 218-223, 1999, doi: 10.1021/ci9800615.

64. Itodo, A.U., Itodo, H.U., "Sorption energies estimation using Dubinin-Radushkevich and Temkin adsorption isotherms", Life Science Journal 7(4), 31-39, 2010.

65. Majer, V., Svoboda, V., Kehiaian, H.V., "Enthalpies of vaporization of organic compounds: a critical review and data compilation", Oxford, Boston: Blackwell Scientific Publications, 1985, ISBN: 9780632015290.

66. Payri, F., Broatch, A., Serrano, J.R., Piqueras, P., "Experimental-theoretical methodology for determination of inertial pressure drop distribution and pore structure properties in wall-flow diesel particulate filters", Energy 36:6731-6744, 2011, doi: 10.1016/j.energy.2011.10.033.

67. Luján, J.M., Bermúdez, V., Piqueras, P., García-Afonso, Ó., "Experimental assessment of pre-turbo aftertreatment configurations in a single stage turbocharged diesel engine. Part 1: Steady-state operation", Energy 80:599-613, 2015, doi: 10.1016/j.energy.2014.05.048.

68. Galindo, J., Serrano, J.R., Arnau, F.J., Piqueras, P., "Highfrequency response of a calculation methodology for gas dynamics based on Independent Time Discretisation", 
Mathematical and Computer Modelling 50 (5):812-822, 2009, doi: 10.1016/j.mcm.2009.05.011.

69. Bissett, E.J., Kostoglou, M., Konstandopoulos, A.G., "Frictional and heat transfer characteristics of flow in square porous tubes of wall-flow monoliths", Chemical Engineering Science 84:255265, 2012, doi:10.1016/j.ces.2012.08.012.

70. Groppi, G., Tronconi, E., "Continuous vs. discrete models of non-adiabatic monolith catalysts", AIChE Journal 42(8):23822387, 1996, doi: 10.1002/aic.690420829.

\section{Contact Information}

Pedro Piqueras, Universitat Politècnica de València, CMT-Motores Térmicos, Camino de Vera s/n, 46022 Valencia, Spain. E-mail: pedpicab@mot.upv.es.

\section{Acknowledgments}

This research has been partially supported by FEDER and the Government of Spain through project TRA2016-79185-R and by the European Union's Horizon 2020 Framework Programme for research, technological development and demonstration under grant agreement number 723976 .

\section{Definitions/Abbreviations}

\section{Acronyms}

$\begin{array}{ll}\text { CFL } & \text { Courant-Friedrichs-Lewy } \\ \text { DOC } & \text { Diesel Oxidation Catalyst } \\ \text { DPF } & \text { Diesel Particulate Filter } \\ \text { ECU } & \text { Electronic Control Unit } \\ \text { EGR } & \text { Exhaust Gas Recirculation } \\ \text { GPF } & \text { Gasoline Particulate Filter } \\ \text { HSDI } & \text { High-Speed Direct Injection } \\ \text { LNT } & \text { Lean NOx Trap } \\ \text { LR-EGR } & \text { Long-Route EGR } \\ \text { OBD } & \text { On-Board Diagnostics } \\ \text { OFA } & \text { Open Frontal Area } \\ \text { PF } & \text { Particulate Filter } \\ \text { PSD } & \text { Particle Size Distribution } \\ \text { RDE } & \text { Real Driving Emissions } \\ \text { SCR } & \text { Selective Catalytic Reduction } \\ \text { SR-EGR } & \text { Short-Route EGR } \\ \text { TWC } & \text { Three-Way Catalyst } \\ \text { VGT } & \text { Variable Geometry Turbine } \\ \text { WLTC } & \text { World harmonized Light vehicles Test Cycle } \\ \end{array}$

\section{Latin letters}

\begin{tabular}{|c|c|}
\hline$a_{i}$ & Constant in shape factor correlation \\
\hline $\mathbf{A}$ & Area \\
\hline$A_{f}$ & Filtration area \\
\hline$b_{i}$ & $\begin{array}{l}\text { Coefficient in } \mathrm{CO} / \mathrm{HC} \text { outlet molar } \\
\text { fraction solution }\end{array}$ \\
\hline$c_{i}$ & $\begin{array}{l}\text { Pressure drop to volumetric flow rate } \\
\text { coefficient }\end{array}$ \\
\hline$c_{\text {gas }}$ & Molar concentration of the gas stream \\
\hline
\end{tabular}

Page 20 of 22

\begin{tabular}{|c|c|c|}
\hline$c_{p}$ & Specific heat & {$[\mathrm{J} / \mathrm{kgK}]$} \\
\hline$C$ & Equivalent thermal capacity & {$[\mathrm{J} / \mathrm{K}]$} \\
\hline$d_{c}$ & Unit collector diameter & {$[\mathrm{m}]$} \\
\hline$d_{c e l l, w}$ & Unit cell diameter & {$[\mathrm{m}]$} \\
\hline$d_{p}$ & Mean pore diameter & {$[\mathrm{m}]$} \\
\hline$d_{\text {part }}$ & Particle diameter & {$[\mathrm{m}]$} \\
\hline$D$ & Diameter & {$[\mathrm{m}]$} \\
\hline$D_{e f}$ & Effective diffusivity & {$\left[\mathrm{m}^{2} / \mathrm{s}\right]$} \\
\hline$D_{K n}$ & Knudsen diffusivity & {$\left[\mathrm{m}^{2} / \mathrm{s}\right]$} \\
\hline$D_{m}$ & Molecular diffusivity & {$\left[\mathrm{m}^{2} / \mathrm{s}\right]$} \\
\hline$D_{\text {part }}$ & Particle diffusion coefficient & {$[-]$} \\
\hline $\boldsymbol{E}_{\boldsymbol{a}}$ & Activation energy & {$[\mathrm{J} / \mathrm{mol}]$} \\
\hline $\boldsymbol{E}_{f}$ & Filtration efficiency & {$[-]$} \\
\hline$f_{s p}$ & Loaded porous wall fraction & {$[-]$} \\
\hline $\boldsymbol{F}_{\boldsymbol{c}}$ & Heat transfer correction coefficient & {$[-]$} \\
\hline$F_{\text {sat }}$ & Porous wall saturation coefficient & {$[-]$} \\
\hline$F_{w}$ & Viscous loss coefficient & {$[-]$} \\
\hline$G_{r, n}$ & Inhibition term of species $n$ in reaction $r$ & {$[-]$} \\
\hline $\boldsymbol{h}$ & Heat transfer coefficient & {$\left[\mathrm{W} / \mathrm{m}^{2} \mathrm{~K}\right.$} \\
\hline $\boldsymbol{H}_{\boldsymbol{f}}$ & Enthalpy of formation & {$[\mathrm{J} / \mathrm{mol}]$} \\
\hline $\boldsymbol{k}$ & Permeability & {$\left[\mathrm{m}^{2}\right]$} \\
\hline $\boldsymbol{k}_{B}$ & Boltzmann constant & {$[\mathrm{J} / \mathrm{K}]$} \\
\hline $\boldsymbol{k}_{m}$ & Mass transfer coefficient & {$[\mathrm{m} / \mathrm{s}]$} \\
\hline $\boldsymbol{k}_{r, \boldsymbol{n}}$ & $\begin{array}{l}\text { Kinetic constant of species } n \text { in } \\
\text { reaction } r\end{array}$ & {$\left[\mathrm{~s}^{-1}\right]$} \\
\hline $\boldsymbol{k}_{\text {reg,n }}$ & $\begin{array}{l}\text { Kinetic constant of soot oxidation by } \\
\text { species } n\end{array}$ & {$[\mathrm{~m} / \mathrm{s}]$} \\
\hline$K$ & Kuwabara’s hydrodynamic factor & {$[-]$} \\
\hline$K_{e q}$ & Equilibrium constant & {$[-]$} \\
\hline $\boldsymbol{K}_{\boldsymbol{i}}$ & Inhibition term coefficient $i$ & {$\left[\mathrm{~s}^{-1}\right]$} \\
\hline Kn & Knudsen number & {$[-]$} \\
\hline$L_{e f}$ & Effective channel length & {$[\mathrm{m}]$} \\
\hline$L_{i n}$ & Inlet channels length & {$[\mathrm{m}]$} \\
\hline$L_{m o n}$ & Monolith length & [m] \\
\hline$L_{\text {out }}$ & Outlet channels length & [m] \\
\hline$m_{s, c e l l}$ & Soot mass in the unit cell & {$[\mathrm{kg}]$} \\
\hline$m_{s, f i l t}$ & Filtrated soot mass & {$[\mathrm{kg}]$} \\
\hline$m_{s, r e g}$ & Regenerated soot mass & {$[\mathrm{kg}]$} \\
\hline$m_{s, s a t_{c e l l}}$ & Saturation mass of the unit cell & {$[\mathrm{kg}]$} \\
\hline $\boldsymbol{m}_{s, w}$ & Soot mass in the porous wall & {$[\mathrm{kg}]$} \\
\hline$\dot{m}$ & Mass flow & {$[\mathrm{kg} / \mathrm{s}]$} \\
\hline$M$ & Molecular weight & {$[\mathrm{kg} / \mathrm{mol}]$} \\
\hline$\dot{\boldsymbol{n}}_{n}$ & Molar flow of species $n$ & {$[\mathrm{~mol} / \mathrm{s}]$} \\
\hline$\dot{n}_{T, g a s}$ & Total exhaust gas mole flow & {$[\mathrm{mol} / \mathrm{s}]$} \\
\hline$N_{i n}$ & Inlet channels number & {$[-]$} \\
\hline$N_{R}$ & Interception parameter & {$[-]$} \\
\hline$p$ & Pressure & {$[\mathrm{Pa}]$} \\
\hline$P_{f}$ & Pre-exponential factor & \\
\hline$P e$ & Peclet number & {$[-]$} \\
\hline$\dot{\boldsymbol{q}}_{h t}$ & Gas to wall heat exchange & {$[\mathrm{W}]$} \\
\hline$\dot{\boldsymbol{q}}_{r}$ & Reaction power & {$[\mathrm{W}]$} \\
\hline$\dot{\boldsymbol{q}}_{r}^{\prime \prime}$ & Reaction power per unit of volume & {$\left[\mathrm{W} / \mathrm{m}^{3}\right]$} \\
\hline
\end{tabular}

$[\mathrm{J} / \mathrm{K}]$

m]

$[\mathrm{m}]$

$[\mathrm{m}]$

$\left[\mathrm{m}^{2} / \mathrm{s}\right]$

$\left.\mathrm{m}^{2} / \mathrm{s}\right]$

[ $/ \mathrm{mol}]$

$\left.\mathrm{W} / \mathrm{m}^{2} \mathrm{~K}\right]$

$\mathrm{J} / \mathrm{mol}]$

[J/K]

$\mathrm{m} / \mathrm{s}$ ]

-]

[m]

[m]

[m]

[m]

$\mathrm{kg}]$

$\mathrm{kg}$

$\mathrm{kg}]$

$\mathrm{kg} / \mathrm{s}]$

$\mathrm{kg} / \mathrm{mol}]$

$\mathrm{mol} / \mathrm{s}]$

mol/s]

[W]

$\left[\mathrm{W} / \mathrm{m}^{3}\right.$ ] 


$\begin{array}{cll}\boldsymbol{Q} & \text { Volumetric flow rate } & {\left[\mathrm{m}^{3} / \mathrm{s}\right]} \\ \boldsymbol{r}_{\boldsymbol{c a t}} & \text { Catalyst particle radius } & {[\mathrm{m}]} \\ \boldsymbol{R} & \text { Equivalent thermal resistance } & {[\mathrm{K} / \mathrm{W}]} \\ \boldsymbol{R}_{\boldsymbol{n}} & \text { Reaction rate of species } n & {\left[\mathrm{~s}^{-1}\right]} \\ \boldsymbol{R} & \text { Universal gas constant } & {[\mathrm{J} / \mathrm{molK}]} \\ \boldsymbol{S}_{\boldsymbol{c}} & \text { Sticking coefficient } & {[-]} \\ \boldsymbol{S} \boldsymbol{C} \boldsymbol{F} & \text { Stokes-Cunningham factor } & {[-]} \\ \boldsymbol{S} \boldsymbol{h} & \text { Sherwood number } & {[-]} \\ \boldsymbol{S}_{\boldsymbol{l}} & \text { Limit saturation coefficient } & {[-]} \\ \boldsymbol{S}_{\boldsymbol{p}} & \text { Geometric surface area } & {\left[\mathrm{m}^{-1}\right]} \\ \boldsymbol{t} & \text { Time } & {[\mathrm{s}]} \\ \boldsymbol{T} & \text { Temperature } & {[\mathrm{K}]} \\ \boldsymbol{u}_{\boldsymbol{i n}} & \text { Inlet flow velocity } & {[\mathrm{m} / \mathrm{s}]} \\ \boldsymbol{u}_{\boldsymbol{o u t}} & \text { Outlet flow velocity } & {[\mathrm{m} / \mathrm{s}]} \\ \boldsymbol{u}_{\boldsymbol{w}} & \text { Filtration velocity } & {[\mathrm{m} / \mathrm{s}]} \\ \boldsymbol{v} & \text { Diffusion volume } & {\left[\mathrm{m}^{3} / \mathrm{mol}\right]} \\ \boldsymbol{V} & \text { Volume } & {\left[\mathrm{m}^{3}\right]} \\ \boldsymbol{w} & \text { Thickness } & {[\mathrm{m}]} \\ \boldsymbol{X}_{\boldsymbol{n}} & \text { Molar fraction of species } n & {[-]} \\ \boldsymbol{Y}_{\boldsymbol{n}} & \text { Mass fraction of species } n & {[-]} \\ \boldsymbol{y} & & \end{array}$

\section{Greek letters}

\begin{tabular}{|c|c|}
\hline$\alpha$ & Clean channel width \\
\hline$\alpha_{n}$ & Completeness index of species $n$ \\
\hline$\Delta p$ & Pressure drop \\
\hline$\Delta H_{a d s / d e s}$ & Adsorption-desorption enthalpy \\
\hline$\varepsilon$ & Emissivity \\
\hline$\varepsilon_{\text {ash }}$ & Ash layer porosity \\
\hline$\varepsilon_{c a t}$ & Catalyst particle porosity \\
\hline$\varepsilon_{p l}$ & Particulate layer porosity \\
\hline$\varepsilon_{w}$ & Loaded porous wall porosity \\
\hline$\varepsilon_{w_{0}}$ & Clean porous wall porosity \\
\hline$\eta_{D}$ & Brownian unit collector efficiency \\
\hline$\eta_{D R}$ & Overall unit collector efficiency \\
\hline$\eta_{g a s, w}$ & Gas to wall heat transfer efficiency \\
\hline$\eta_{\text {int }}$ & Internal pore diffusion efficiency \\
\hline$\eta_{R}$ & Interception unit collector efficiency \\
\hline$\theta$ & Surface coverage \\
\hline$\Theta_{w c}$ & Washcoat fraction \\
\hline $\boldsymbol{\kappa}$ & Conductivity \\
\hline$\lambda$ & Gas mean free path \\
\hline $\boldsymbol{\mu}$ & Gas dynamic viscosity \\
\hline$v_{n}$ & Stoichiometric coefficient of species $n$ \\
\hline$\xi$ & Particulate layer corrector thickness \\
\hline$\rho$ & Density \\
\hline$\rho_{s, w}$ & Soot packing density \\
\hline$\sigma$ & Cell density \\
\hline$\sigma_{s b}$ & Stefan-Boltzman constant \\
\hline$S_{c a t}$ & Catalyst pressure drop coefficient \\
\hline$\varsigma_{t}$ & Inertial pressure drop coefficient \\
\hline$\tau$ & Residence time \\
\hline
\end{tabular}

[m]

[-]

[Pa]

[J/mol]

[-]

[-]

[-]

[-]

[-]

[-]

[-]

[-]

[-]

[-]

[-]

[-]

[-]

[W/mK]

[m]

[ $\mathrm{kg} / \mathrm{ms}]$

[-]

[m]

$\left[\mathrm{kg} / \mathrm{m}^{3}\right]$

$\left[\mathrm{kg} / \mathrm{m}^{3}\right]$

$\left[\mathrm{m}^{-2}\right]$

$\left[\mathrm{W} / \mathrm{m}^{2} \mathrm{~K}^{4}\right]$

[-]

[-]

[s]

$\begin{array}{cll}\boldsymbol{\varphi} & \text { Percolation factor } & {[-]} \\ \boldsymbol{\phi} & \text { Thiele modulus } & {[-]} \\ \boldsymbol{\Phi}_{\boldsymbol{\rho}_{\boldsymbol{s}}} & \text { Soot density factor } & {[-]} \\ \boldsymbol{\chi} & \text { Shape factor } & {[-]} \\ \boldsymbol{\psi} & \text { Specific storage capacity } & {[-]} \\ \boldsymbol{\Psi}_{\boldsymbol{T}, \boldsymbol{a}-\boldsymbol{s}} & \text { Maximum moles adsorpted in active } & {\left[\mathrm{mol} / \mathrm{m}^{3}\right]} \\ & \text { site } a-s & \\ \boldsymbol{\omega} & \text { Meso-pores tortuosity in catalyst } & {[-]} \\ & \text { particles } & \end{array}$

Subscripts

\begin{tabular}{|c|c|}
\hline$a-s$ & Active site \\
\hline$a d s$ & Adsorption \\
\hline ash & Ash layer \\
\hline$a x$ & Axial \\
\hline$c$ & Conduction \\
\hline can & Canning \\
\hline cat & Catalyst \\
\hline ch & Monolith channel \\
\hline des & Desorption \\
\hline$e f$ & Effective \\
\hline ext & External \\
\hline$f r$ & Friction \\
\hline gap & Gap between mat and canning \\
\hline gas & Exhaut gas flow \\
\hline in & Inlet \\
\hline int & Internal \\
\hline $\lim$ & Limit \\
\hline mat & Canning mat \\
\hline mon & Monolith \\
\hline$n$ & Species \\
\hline out & Outlet \\
\hline$o x$ & Oxidation \\
\hline$p l$ & Particulate layer \\
\hline$p l_{\text {hom }}$ & Homogenous particulate layer \\
\hline post & Related to a device downstream of the monolith \\
\hline pre & Related to a device upstream of the monolith \\
\hline$r$ & Radiation \\
\hline rad & Radial \\
\hline$s$ & Soot \\
\hline$s p$ & Soot penetration into porous wall \\
\hline subs & Substrate \\
\hline sur & Surface \\
\hline$w$ & Substrate wall \\
\hline$w_{0}$ & Clean porous wall \\
\hline$w c$ & Washcoat \\
\hline
\end{tabular}

\section{Superscripts}

$\begin{array}{ll}\boldsymbol{B} & \text { Channels beam } \\ \boldsymbol{m} & \text { Reaction order } \\ \boldsymbol{p} & \text { Time step identifier }\end{array}$




\section{Appendix. Lumped heat transfer sub-model parameters}

According to Figure 8, an equivalent thermal resistance $R_{g a s, w}$ governs the gas to wall heat transfer in monolith channels. Non-linear onedimensional dependence is characteristic in the gas and wall temperature profile as well as in heat transfer coefficient, especially in wall-flow monoliths [69]. To avoid this concern in lumped modelling, a heat exchanger efficiency is defined, thus expressing $R_{g a s, w}$ as:

$$
R_{g a s, w}=\frac{1}{\eta_{g a s, w} c_{p} \dot{m}_{c h}}
$$

The thermal inertia of the substrate is also taken into account by means of the thermal capacity $\left(C_{w}\right)$, whose definition assumes channel walls as plane sheets. Thus in flow-through monoliths it is computed as

$$
C_{w, \text { flow-through }}=4\left(\alpha+\frac{w_{w}}{2}\right) L_{m o n} \frac{w_{w}}{2} \rho_{w} c_{p_{w}}
$$

while in wall-flow monoliths the impact of the particulate matter layer is also added:

$$
C_{w, \text { wall-flow }}=4\left(\alpha+\frac{w_{w}}{2}\right) L_{m o n} w_{w} \rho_{w} c_{p_{w}}+4\left(\alpha-w_{p l}\right) w_{p l} L_{e f} \rho_{p l} c_{p_{p l}}
$$

The radial variation of the substrate wall temperature is governed by the radial conduction from the monolith core to the external surface by means of the equivalent thermal resistance $R_{r a d, c}$ as discussed in [17]. It sets the heat transfer towards the monolith external surface:

$$
\begin{aligned}
& R_{\text {rad }, c}^{B, B-1}=\ln \left(\frac{D_{\text {ext }}^{B}}{D_{\text {int }}^{B-1}}\right) \frac{1}{2 \pi L_{e f} \kappa_{\text {rad }}} F_{c} \\
& R_{\text {rad }, c}^{B, B+1}=\ln \left(\frac{D_{\text {ext }}^{B+1}}{D_{\text {int }}^{B}}\right) \frac{1}{2 \pi L_{e f} \kappa_{\text {rad }}} F_{c}
\end{aligned}
$$

In Eqs. (84) and (85) superscript $B$ identifies the channels beam in case of radial discretization, $F_{c}$ is a correction coefficient to account for pipes to honeycomb monolithic structures [17] and $D_{\text {ext }}^{B}$ and $D_{\text {int }}^{B}$ are the external and internal diameters of the channel beam $B$. The radial conductivity $\left(k_{\text {rad }}\right)$ is obtained from the porous substrate conductivity applying the continuum approximation of heat conduction proposed by Groppi and Tronconi [70] for the unit cell of honeycomb monolith reactors. Neglecting the influence of a particulate matter layer [17], the radial conductivity of either a flow-through or a wall-flow monolith is expressed as:

$$
\kappa_{\text {rad }}=\left(\frac{w_{w}}{\kappa_{\text {subs }}\left(\alpha+w_{w}\right)}+\frac{\alpha}{\kappa_{\text {gas }} \alpha}\right)^{-1}
$$

Similarly, the surface temperature in the external canning can be also predicted accounting for its capacitance and all the involved thermal equivalent resistances, which are depicted in Figure 8(b). Applying cylindrical coordinates to this region, these parameters are defined as

$$
\begin{gathered}
C_{\text {sur }_{i}}=L_{\text {ef }} \frac{\pi}{4} \sum \rho_{j} c_{p_{j}}\left(D_{j_{\text {ext }}}^{2}-D_{j_{\text {int }}}^{2}\right) \\
R_{j, c}=\frac{\ln \left(\frac{D_{j_{\text {ext }}}}{D_{j_{\text {int }}}}\right)}{2 \pi L_{\text {mon }} \kappa_{j}} \quad(88) \\
R_{\text {gap }, r}=\frac{\frac{1}{\varepsilon_{\text {mat }}}+\frac{D_{\text {gap }, \text { int }}}{D_{\text {gap }, \text { ext }}}\left(\frac{1}{\varepsilon_{\text {can }}}-1\right)}{\sigma_{\text {sb }} \pi L_{\text {mon }} D_{\text {int }} \frac{T_{\text {sur }, \text { int }}^{4}-T_{\text {sur }, \text { int }}-T_{\text {sur }, \text { ext }}}{T_{\text {sur }}}=R_{\text {mat }, c}+\frac{R_{\text {gap }, c} R_{\text {gap }, r}}{R_{\text {gap }, c}+R_{\text {gap }, r}}+R_{\text {can }, c}} \\
R_{\text {ext }}=\frac{1}{\pi D_{\text {ext }} L_{\text {mon }} h_{\text {ext }}}
\end{gathered}
$$

where subscript $i$ identifies the external and internal nodes and $j$ every layer (mat, gap and metal can). In thermal equivalent resistances, $c$ is referred to conduction and $r$ to radiation. All the parameters are assumed constant during the time-step according to the flow, substrate and canning operating conditions. In particular, the radiation terms are computed making use of the temperature values at the beginning of every time-step. This assumption allows obtaining an explicit solution of the wall temperature. The impact on the solution is negligible due to the difference in several orders of magnitude between the characteristic time of the thermal transient and the time-step. 